Research Article

\title{
Three-Dimensional High-Resolution MIMO Radar Imaging via OFDM Modulation and Unitary ESPRIT
}

\author{
Jingjing Zhao $(\mathbb{D}$, Yongxiang Liu $(\mathbb{D}$, Kai Huo $\mathbb{D}$, Jiaxi Ye, and Bo Xiao \\ College of Electronic Science and Engineering, National University of Defense Technology, Changsha, China \\ Correspondence should be addressed to Yongxiang Liu; lyx_bible@sina.com
}

Received 18 March 2020; Revised 12 May 2020; Accepted 25 May 2020; Published 27 June 2020

Academic Editor: Ramon Sancibrian

Copyright (C) 2020 Jingjing Zhao et al. This is an open access article distributed under the Creative Commons Attribution License, which permits unrestricted use, distribution, and reproduction in any medium, provided the original work is properly cited.

\begin{abstract}
Imaging and recognition of targets with complex maneuvers bring a new challenge to conventional radar applications. In this paper, the three-dimensional (3D) high-resolution image is attained in real-time by a Multiple-Input-Multiple-Output (MIMO) radar system with single Orthogonal-Frequency-Division-Multiplexing (OFDM) pulse. First, to build the orthogonal transmit waveform set for MIMO transmission, we utilize complex orthogonal designs (CODs) for OFDM subcarrier modulation. Based on the OFDM modulation, a preprocessing method is developed for transmit waveform separation without conventional matched filtering. The result array manifold is the Kronecker product of the steering vectors of subcarrier/transmit antenna/receive antenna uniform linear arrays (ULAs). Then, the high-resolution image of target is attained by the Multidimensional Unitary Estimation of Signal Parameters via Rotational Invariant Techniques (MD-UESPRIT) algorithm. The proposed imaging procedures include the multidimensional spatial smoothing, the unitary transform via backward-forward averaging, and the joint eigenvalue decomposition (JEVD) algorithm for automatically paired coordinates estimation. Simulation tests compare the reconstruction results with the conventional methods and analyze the estimation precision relative to signal-to-noise ratio (SNR), system parameters, and errors.
\end{abstract}

\section{Introduction}

In the past few years, the OFDM waveform has been demonstrated as a potential radar waveform [1-8]. An OFDM waveform consists of multiple subcarriers which are mathematically orthogonal in the time domain. Each subcarrier could be modulated by a phase code sequence; hence, an OFDM pulse [5] has high spectral efficiency as linear frequency modulation (LFM) pulse, along with a bit-to-bit diversity as the phase-coded pulse. Besides, the OFDM signal also offers frequency diversity to the radar system. These properties make OFDM signals particularly suitable for full orthogonal MIMO radar applications. According to the allocation schemes of multiple subcarriers, there were different applications. A step frequency technique was adopted $[9,10]$ that the orthogonality is fulfilled, but the range resolution is limited. Interleaved OFDM (I-OFDM) was proposed and taken in [11-16]. An equidistant and exclusive subset of subcarriers is assigned to each transmit antenna. In this way, the range resolution is preserved; however, the maximum unambiguous range interval is reduced. A novel non-equidistant subcarrier interleaving approach $[17,18]$ was adopted to attain a full unambiguously measurable range. However, lacking -some subcarriers may lead to an increase inthe sidelobe level. In $[16,19,20]$, the complete set of subcarriers were allocated to each transmit antenna, whereas, on each subcarrier, complex orthogonal designs (CODs) [21-23] were adopted for multiple antennas to ensure orthogonality.

The three-dimensional (3D) radar imaging provides more detailed and accurate descriptions of the target and is meaningful for feature extraction and identification. Along with the Inverse Synthetic Aperture Radar (ISAR) technique, Interferometric ISAR (In-ISAR) [24-26] and MIMO-ISAR [27-29] techniques have to cope with the complex motion compensation, especially for maneuvering targets. Instead of the time sampling in ISAR, MIMO radar systems utilize spatial sampling. Moreover, with an orthogonal transmit set, 
the equivalent virtual array could be formed in a single snapshot illumination with fewer elements than a conventional array radar. MIMO radar 3D imaging techniques $[12,14,30-38]$ have been proposed in recent years. In $[12,14,30,31,38]$, with wideband transmission for range resolution, the two-dimensional (2D) cross ranges were resolved with two mutually perpendicular uniform linear arrays (ULAs). Additional range unit alignment and compensation steps were still necessary before cross-range imaging. In $[32,33]$, assuming all scatterers fall into the same range bin for narrowband radar configuration, the $3 \mathrm{D}$ images were reconstructed with two bistatic antenna arrays. The range alignment was then avoided, but more antennas were needed compared to the wideband case. Sparse arrays $[34,36,37]$ were utilized to solve the problem, and sparsity recovery-based methods were proposed for high-resolution imaging.

The OFDM-MIMO radar configuration is applied in this paper to reduce antennas used for 3D imaging and remove the range alignment steps. We follow the design criterion in [20] that CODs are adopted for subcarrier coding. The uniformly spaced subcarriers form a ULA in the frequency domain for radial-range estimation. The transmit/receive antenna arrays are two ULAs which are located mutually perpendicular and form an $L$-shape to obtain $2 \mathrm{D}$ cross-range resolution. At each receiver, other than the matched filtering set for waveform separation and pulse compression, we apply Fast Fourier Transform (FFT) to the echo for subcarriers separation, and the frequency domain echoes from different transmit antennas could be separated via decoding. The demodulated results of the subcarrier/transmitter/receiver arrays are stored for 3D coordinate estimation. Therefore, no range alignment is needed.

To break the Rayleigh criterion of minimum resolvable separation, we present a 3D imaging method based on the Estimation of Signal Parameters via Rotational Invariant Techniques (ESPRIT) algorithm. The ESPRIT algorithm is a commonly used high-resolution parameter estimation method [39-41]. It exploits the rotational invariance among the signal subspace and has no searching process as in the MUltiple SIgnal Classification (MUSIC) techniques. The high-resolution imaging method based on the ESPRIT algorithm was reported in [33, 36, 38, 42-46]. However, three problems have to be taken into account for the ESPRIT algorithm applied in OFDM-MIMO radar imaging. First, the preprocessed data are only one snapshot available that the frequency domain echoed from different scatterers is coherent, and the covariance matrix is no longer full rank. This leads to the failure of the ESPRIT algorithm. Second, for the 3D-ESPRIT estimation, the matrix size is usually large that the complex-valued eigenvalue decomposition (EVD) or singular value decomposition (SVD) is computationally expensive. Moreover, the standard ESPRIT algorithm does not take the utmost of the complex conjugate of the initial data. Finally, the extra pairing procedure is required for 3D reconstruction. The first aforementioned problem could be solved by applying a multidimensional spatial smoothing technique to restore the covariance matrix to full rank. For the second one, involving the backward-forward averaging method and unitary transformation, we apply a 3D UnitaryESPRIT (3D-UESPRIT) algorithm. Although the snapshot number is doubled after backward-forward averaging, the computational burden is eased since the algorithm involves only real-valued computations afterwards. Last but not least, the joint eigenvalue decomposition (JEVD) [47-51] method is adopted for $3 \mathrm{D}$ estimation to diagonalize the three matrix pencils simultaneously, which leads to the $3 \mathrm{D}$ coordinates estimation results automatically paired.

In this paper, we first establish an OFDM-MIMO radar imaging model based on OFDM's multicarrier structure; the echo is then preprocessed to construct the data vector that suits the ESPRIT form. Multidimensional spatial smoothing and backward-forward averaging techniques are introduced to build the multisnapshots data matrix. Finally, the superresolution images are obtained by exploiting the shift-invariance property with only real-valued computations. Imaging results of the 2D EM computed data for an Airbus A320 plane and the 3D simulated data for the point scatterer model of a Boeing 777 plane are depicted and compared to prove the efficiency of our proposed imaging methods for OFDM-MIMO radar. The proposed algorithm provides better reconstruction precision compared with the conventional Fourier-based method and has lower computational complexity than the MD-MUSIC and MD-ESPRIT algorithms [42]. Reconstruction accuracy is then analyzed corresponding to the system parameters and system errors for further optimization.

Notations: $(\cdot)^{\mathrm{T}},(\cdot)^{\mathrm{H}},(\cdot)^{*},(\cdot)^{-}$, and $(\cdot)^{\dagger}$ denote transpose, conjugate transpose, conjugate, inverse operation, and pseudoinverse operation, respectively. The bolded capital letters and lowercase letters represent the matrices and vectors, $\|\mathbf{a}\|$ denotes the modulus of the vector, and $\|\mathbf{A}\|$ is the norm of the matrix. $\otimes, \odot$, and ${ }^{\circ}$ are the Kronecker product, Hadamard product, and Khatri-Rao product, respectively. $\operatorname{Diag}\{\mathbf{a}\}$ is a diagonal matrix whose diagonal is the vector $\mathbf{a}$, whereas $\operatorname{diag}\{\mathbf{A}\}$ is the column vector of the matrix $\mathbf{A} . \mathfrak{R}(\cdot)$ and $\mathfrak{J}(\cdot)$ denote the real and the imaginary part, respectively.

\section{Signal Model and Preprocessing Method}

In this section, we briefly introduce an OFDM-MIMO system configuration for $3 \mathrm{D}$ imaging, then develop the received signal model, and propose a preprocessing method for transmit waveform separation and imaging center compensation.

2.1. OFDM-MIMO System and Echo Model. In the MIMO radar system, $P$ transmit and $Q$ receive antennas form two ULAs. As shown in Figure 1, the transmit array is parallel to the $x$-axis and uniformly spaced by $d_{t}$; that is, coordinate of the $p$-th transmit antenna is $\mathbf{t}_{p}=\mathbf{t}_{0}+\left[p d_{t}, 0,0\right]^{\mathrm{T}}=$ $t_{0}+p d_{t} \mathbf{x}$, where $p=0, \ldots, P-1$ indicates the transmit antenna index, $\mathbf{t}_{0}=\left[x_{t, 0}, y_{t, 0}, z_{t, 0}\right]^{\mathrm{T}}$ denotes the start of transmit array which is adjacent to the coordinate system center $\mathbf{o}_{r}$, and $\mathbf{x}=[1,0,0]^{\mathrm{T}}$ is the unit vector of $x$-axis. Similarly, the receive array is parallel to $y$-axis with the space 


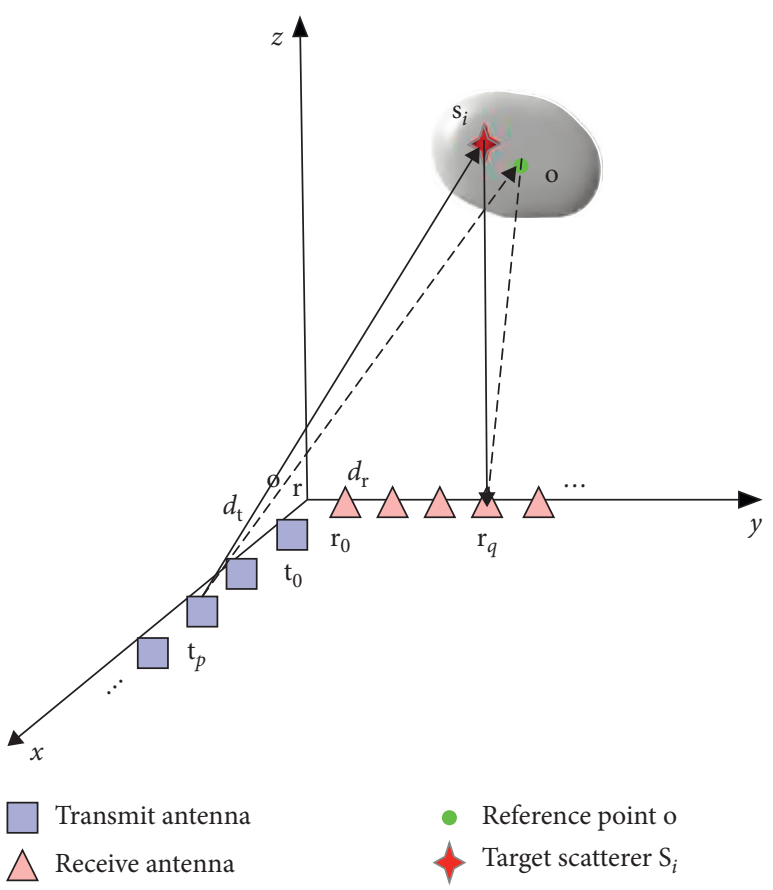

Figure 1: MIMO radar system configuration.

being $d_{r}, q$-th of which is denoted by $\mathbf{r}_{q}=r_{0}+q d_{r} \mathbf{y}$, where $\mathbf{r}_{0}=\left[x_{r, 0}, y_{r, 0}, z_{r, 0}\right]^{\mathrm{T}}$ and $\mathbf{y}=[0,1,0]^{\mathrm{T}}$.

Each transmit element in the radar system emits an OFDM signal modulated at the initial carrier frequency $f_{0}$. The baseband OFDM signal consists of $N$ mutually orthogonal subcarriers with a uniform frequency spacing $\Delta f$; hence, the total bandwidth is $B w=N \Delta f$. The $n$-th subcarrier is modulated with a $K$-bit coding sequence $\mathbf{c}_{n, p}=\left[a_{n, p}^{(0)}, \ldots, a_{n, p}^{(K-1)}\right]^{\mathrm{T}}$. Complex orthogonal designs (CODs) [21-23] which fulfill

$$
\mathbf{c}_{n, p}^{H} \mathbf{c}_{n, p^{\prime}}= \begin{cases}1, & p=p^{\prime}, \\ 0, & p \neq p^{\prime},\end{cases}
$$

are applied [20] to build the orthogonal waveform set. Construct the matrix $\mathbf{C}_{n}=\left[\mathbf{c}_{n, 0}, \ldots, \mathbf{c}_{n, P-1}\right] \in \mathbb{C}^{K \times P}$ and rewrite (1) as

$$
\mathbf{C}_{n}^{H} \mathbf{C}_{n}=\mathrm{I}_{P}
$$

The bit width $t_{b}$ meets the orthogonal condition that $t_{b} \Delta f=1$. As a cyclic prefix (CP), tail of the original OFDM bit is added at the head of each transmitted OFDM symbol to remove the Intersymbol Interference (ISI) [7, 20]. Thus, the symbol duration is $t_{s}=t_{b}+t_{c}$, with the CP duration being $t_{c}=\alpha t_{b}$. The $p$-th baseband OFDM pulse is given by

$$
u_{p}(t)=\sum_{n=0}^{N-1} \sum_{k=0}^{K-1} a_{n, p}^{(k)} \exp \left\{j 2 \pi n \Delta f\left(t-(k+1) t_{c}\right)\right\} \operatorname{rect}\left(\frac{t-k t_{s}}{t_{s}}-\frac{1}{2}\right) \text {, }
$$

where $p=0, \ldots, P-1$ and $\operatorname{rect}(x)= \begin{cases}1, & -1 / 2 \leq x<1 / 2, \\ 0, & \text { otherwise }\end{cases}$ is the rectangular window. The baseband signal $u_{p}(t)$ is then modulated at the initial carrier frequency $f_{0}$ for transmission.

Consider a far-filed target composed of $I$ ideal point-like scatterers, the echo model is given. The $i$-th scatterer coordinate is $\mathbf{s}_{i}=\left[x_{i}, y_{i}, z_{i}\right]^{\mathrm{T}}$, and the relative scattering amplitude is $\sigma_{i}$, which is a constant within an OFDM pulse duration and for multiple transmit/receive paths. Suppose the target velocity has been fully estimated and compensated before preprocessing [7], the Doppler effect is ignored in the echo model. At the $q$-th $(q=0, \ldots, Q-1)$ receive antenna, let $t=\tau_{\text {min }}+k t_{s}+t_{c}+t_{0}$, with $\tau_{\text {min }}$ being the start of the sampling window and $t_{0} \in\left[0, t_{b}\right)$. The relative round-way delay of the $(p, q)$-th transmit/receive path and $i$-th scatterer is

$$
\tau_{p, q}^{(i)}=\frac{\left(\left\|\mathbf{s}_{i}-\mathbf{t}_{p}\right\|+\left\|\mathbf{r}_{q}-\mathbf{s}_{i}\right\|\right)}{c-\tau_{\min }}
$$

where $c$ is the velocity of light; then the echo after downconversion is expressed as

$$
x_{q}^{(k)}\left(t_{0}\right)=\sum_{p=0}^{P-1} \sum_{i=1}^{I} \sigma_{i} \exp \left(-j 2 \pi f_{0} \tau_{p, q}^{(i)}\right) \sum_{n=0}^{N-1} \exp \left\{j 2 \pi n \Delta f\left(t_{0}-\tau_{p, q}^{(i)}\right)\right\} \cdot \sum_{k^{\prime}=0}^{K-1} a_{n, p}^{\left(k^{\prime}\right)} \operatorname{rect}\left(\frac{\left(k-k^{\prime}\right) t_{\mathrm{s}}+t_{\mathrm{c}}+t_{0}-\tau_{p, q}^{(i)}}{t_{\mathrm{s}}}-\frac{1}{2}\right)+w_{q}^{(k)}\left(t_{0}\right),
$$

where $w_{q}^{(k)}\left(t_{0}\right)$ is the noise term, which is the zero-mean complex Additive White Gaussian Noise (AWGN). Assume $\tau_{p, q}^{(i)} \in\left[0, t_{c}\right), \forall p, q, i$; then, the window function goes as

$$
\operatorname{rect}\left(\frac{\left(k-k^{\prime}\right) t_{s}+t_{c}+t_{0}-\tau_{p, q}^{(i)}}{t_{s}}-\frac{1}{2}\right)=\delta\left(k-k^{\prime}\right)
$$

where $\delta(\cdot)$ is the impulse function. Hence, there is no intersymbol interference (ISI) in the echo. Furthermore, set the sampling frequency as $f_{s}=B w=N \Delta f$; thus, $t_{0}=l t_{b} / N,(l=0, \ldots, N-1)$; the discrete-time echo is written as 


$$
x_{l, q}^{(k)}=\sum_{n=0}^{N-1} \exp \left(j 2 \pi \frac{n l}{N}\right) \sum_{p=0}^{P-1} a_{n, p}^{(k)} \sum_{i=1}^{I} \exp \left\{-j 2 \pi\left(f_{0}+n \Delta f\right) \tau_{p, q}^{(i)}\right\} \sigma_{i}+w_{l, q}^{(k)}
$$

where $x_{l, q}^{(k)}$ and $w_{l, q}^{(k)}$ denote the sampled echo and noise term, respectively.
2.2. Preprocessing Method. At the $q$-th receive element, for the $k$-th OFDM bit, compute the DFT of (7) towards the fasttime sampling index $l$; the frequency-domain echo is denoted by

$$
X_{n, q}^{(k)}=\frac{1}{N} \sum_{l=0}^{N-1} \exp \left(-j 2 \pi \frac{n l}{N}\right) x_{l, q}^{(k)}=\sum_{p=0}^{P-1} a_{n, p}^{(k)} \sum_{i=1}^{I} \exp \left\{-j 2 \pi\left(f_{0}+n \Delta f\right) \tau_{p, q}^{(i)}\right\} \sigma_{i}+W_{n, q}^{(k)}
$$

where $X_{n, q}^{(k)}$ is the $n$-th point of the DFT results and also denotes frequency-domain echo on the $n$-th subcarrier and $W_{n, q}^{(k)}$ is the frequency-domain noise. $N$ subcarriers are separated without any Intercarrier Interference (ICI) in (8), but echoes from $P$ transmit antennas are still mixed. For transmit separation and subcarrier decoding, for each subcarrier index $n$, we stack the DFT results of $K$ bits in a column vector $\mathbf{x}_{n, q}=\left[X_{n, q}^{(0)}, \ldots, X_{n, q}^{(K-1)}\right]^{\mathrm{T}}$ and give the matrix form expression of $\mathbf{x}_{n, q}$ as

$$
\mathbf{x}_{n, q}=\mathbf{C}_{n} \mathbf{B}_{n, q} \boldsymbol{\sigma}+\mathbf{w}_{n, q} \in \mathbb{C}^{K \times 1},
$$

with $\mathbf{C}_{n} \in \mathbb{C}^{K \times P}$ defined in (2) and

$$
\mathbf{B}_{n, q}=\left[\begin{array}{ccc}
\beta_{n, 0, q}^{(1)} & \cdots & \beta_{n, 0, q}^{(I)} \\
\vdots & \ddots & \vdots \\
\beta_{n, P-1, q}^{(1)} & \cdots & \beta_{n, P-1, q}^{(I)}
\end{array}\right] \in \mathbb{C}^{P \times I},
$$

where the $(p, i)$-th element

$$
\beta_{n, p, q}^{(i)}=\exp \left\{-j 2 \pi\left(f_{0}+n \Delta f\right) \tau_{p, q}^{(i)}\right\}
$$

is the channel response of the $i$-th scatterer, on $n$-th subcarrier, for the $(p, q)$-th transmit/receive path, and $\sigma=\left[\sigma_{1}, \ldots, \sigma_{I}\right]^{\mathrm{T}} \in \mathbb{C}^{I \times 1} \quad$ and $\mathbf{w}_{n, q}=\left[W_{n, q}^{(0)}, \ldots, W_{n, q}^{(K-1)}\right]^{\mathrm{T}} \in \mathbb{C}^{K \times 1}$ are the scattering coefficient vector and the frequency-domain noise vector, respectively.

Based on (1), left multiplying (9) with $\mathbf{C}_{n}^{H}$ yields

$$
\begin{aligned}
\mathbf{y}_{n, q} & =\mathbf{C}_{n}^{H} \mathbf{x}_{n, q}=\mathbf{B}_{n, q} \boldsymbol{\sigma}+w_{n, q}^{\prime} \\
& =\left[y_{n, 0, q}, \ldots, y_{n, P-1, q}\right]^{\mathrm{T}} \in \mathbb{C}^{P \times 1},
\end{aligned}
$$

where $y_{n, p, q}$ denoted the demodulated echo and $\mathbf{w}_{n, q}^{\prime}$ is the modified noise vector without change of power. Clearly, the $P$ transmit waveforms are separated in $\mathbf{y}_{n, q}$.

As is shown in Figure 1, let $\mathbf{o}=\left[x_{0}, y_{0}, z_{0}\right]^{\mathrm{T}}$ be the center of the imaging area, let $d_{0}=\|\mathbf{o}\|$ be the radial distance, and let $\mathbf{d}_{0}=\mathbf{o} / d_{0}$ be the unit vector of the radar Line of Sight (LOS). For each OFDM subcarrier and each transmit/receive pair, we define the following compensation term:

$$
\beta_{n, p, q}^{(0)}=\exp \left\{j 2 \pi\left(f_{0}+n \Delta f\right) \tau_{p, q}^{(0)}\right\},
$$

where $\tau_{p, q}^{(0)}=\left(\left\|\mathbf{o}-\mathbf{t}_{p}\right\|+\left\|\mathbf{r}_{q}-\mathbf{o}\right\|\right) / c-\tau_{\min } \cdot y_{n, p, q}$ is multiplied with $\beta_{n, p, q}^{(0)}$ for initial phase compensation, and

$$
\begin{aligned}
s_{n, p, q}= & \beta_{n, p, q}^{(0)} y_{n, p, q}=\sum_{i=1}^{I} \exp \left\{j 2 \pi\left(f_{0}+n \Delta f\right)\left(\tau_{p, q}^{(0)}-\tau_{p, q}^{(i)}\right)\right\} \sigma_{i} \\
& +\widetilde{w}_{n, p, q},
\end{aligned}
$$

where $\widetilde{w}_{n, p, q}$ denotes the noise after compensation, the power of which is also unchanged. Since $\tau_{p, q}^{(0)}-\tau_{p, q}^{(i)}=$ $\left(\left\|\mathbf{o}-\mathbf{t}_{p}\right\|+\left\|\mathbf{r}_{q}-\mathbf{o}\right\|-\left\|\mathbf{s}_{i}-\mathbf{t}_{p}\right\|-\left\|\mathbf{r}_{q}-\mathbf{s}_{i}\right\|\right) / c$, following the lemma in [31] and ignoring the quadratic and higher order terms,

$$
\begin{aligned}
\left\|\mathbf{o}-\mathbf{t}_{p}\right\|+\left\|\mathbf{r}_{q}-\mathbf{o}\right\|-\left\|\mathbf{s}_{i}-\mathbf{t}_{p}\right\|-\left\|\mathbf{r}_{q}-\mathbf{s}_{i}\right\| \\
\approx-2 \overline{\mathbf{s}}_{i}^{\mathrm{T}} \mathbf{d}_{0}+\frac{1}{d_{0}}\left[\overline{\mathbf{s}}_{i}-\left(\overline{\mathbf{s}}_{i}^{\mathrm{T}} \mathbf{d}_{0}\right) \mathbf{d}_{0}\right]\left(\mathbf{t}_{p}+\mathbf{r}_{q}\right) \\
=-2 u_{i}+\frac{1}{d_{0}}\left(p d_{\mathrm{t}} \tilde{x}_{i}+q d_{\mathrm{r}} \tilde{y}_{i}+\xi_{i}\right),
\end{aligned}
$$

where $\overline{\mathbf{s}}_{i}=\mathbf{s}_{i}-\mathbf{o}, u_{i}=\overline{\mathbf{s}}_{i}^{T} \mathbf{d}_{0}$ denotes the radial range, which is the projection of vector $\overline{\mathbf{s}}_{i}$ on the direction of radar LOS $\mathbf{d}_{0}$, and $\mathbf{v}_{i}=\overline{\mathbf{s}}_{i}-\left(\overline{\mathbf{s}}_{i}^{T} \mathbf{d}_{0}\right) \mathbf{d}_{0} \doteq\left(\widetilde{x}_{i}, \widetilde{y}_{i}, \widetilde{z}_{i}\right)^{\mathrm{T}}$ is the residual vector of $\overline{\mathbf{s}}_{i}$ with respect to the radar LOS component. Then $\tilde{x}_{i}=\mathbf{v}_{i}^{\mathrm{T}} \mathbf{x}$ and $\tilde{y}_{i}=\mathbf{v}_{i}^{T} \mathbf{y}$ are $2 \mathrm{D}$ cross-range coordinates, and $\xi_{i}=\mathbf{v}_{i}^{\mathrm{T}}\left(\mathbf{t}_{0}+\right.$ $\mathbf{r}_{0}$ ) is the initial range term with respect to starts of the transmit/receive ULAs. Conversely, the scatterer coordinates could be reconstructed with $\left(u_{i}, \tilde{x}_{i}, \tilde{y}_{i}\right)$ by

$$
\left\{\begin{array}{l}
\widehat{x}_{i}=\tilde{x}_{i}+u_{i} \mathbf{d}_{0}[1]+x_{0}, \\
\widehat{y}_{i}=\tilde{y}_{i}+u_{i} \mathbf{d}_{0}[2]+y_{0}, \\
\widehat{z}_{i}=\frac{u_{i}-\left(\hat{x}_{i}-x_{0}\right) \mathbf{d}_{0}[1]-\left(\hat{y}_{i}-y_{0}\right) \mathbf{d}_{0}[2]}{\mathbf{d}_{0}[3]}+z_{0} .
\end{array}\right.
$$

With the narrowband and far-filed assumption, that is, $f_{0} \gg B w$ and $\tilde{x}_{i}, \tilde{y}_{i}, d_{t}, d_{r} \ll d_{0}$, ignore cross-range offsets on multiple subcarriers and rewrite (14) as 


$$
s_{n, p, q} \approx \sum_{i=1}^{I} \tilde{\sigma}_{i} \exp \left(-j 2 \pi n \frac{u_{i}}{N \Delta u}\right) \exp \left\{j 2 \pi p \frac{\tilde{x}_{i}}{P \Delta \widetilde{x}}\right\} \exp \left\{j 2 \pi q \frac{\tilde{y}_{i}}{Q \Delta \tilde{y}}\right\}=\sum_{i=1}^{I} \widetilde{\sigma}_{i} e^{j n \omega_{1, i}} e^{j p \omega_{2, i}} e^{j q \omega_{3, i}}+\widetilde{w}_{n, p, q},
$$

where $\tilde{\sigma}_{i}=\sigma_{i} \exp \left\{j 2 \pi\left(-2 u_{i}+\xi_{i}\right) / \lambda\right\}$ denotes the phase modulated scattering amplitude, which is also constant for multiple transmit/receive paths,

$$
\left\{\begin{array}{l}
\Delta u=\frac{c}{2 N \Delta f}=\frac{c}{2 B w}, \\
\Delta \widetilde{x}=\frac{c d_{0}}{f_{0} P d_{t}}=\frac{\lambda_{0} d_{0}}{L_{t}}, \\
\Delta \tilde{y}=\frac{c d_{0}}{f_{0} Q d_{r}}=\frac{\lambda_{0} d_{0}}{L_{r}},
\end{array}\right.
$$

which are the resolutions of the radial/cross ranges, respectively, with $\lambda_{0}=c / f_{0}$ being the system wavelength and $L_{t}=P d_{t}$ and $L_{r}=Q d_{r}$ denoting the transmit/receive array lengths. Therefore, $\left\{s_{n, p, q}\right\}$ is the uniformly spaced sampling in the $3 \mathrm{D}$ spatial spectrum with

$$
\left\{\begin{array}{l}
\omega_{1, i}=\frac{-2 \pi u_{i}}{(N \Delta u)} \\
\omega_{2, i}=\frac{2 \pi \tilde{x}_{i}}{(P \Delta \tilde{x})} \\
\omega_{3, i}=\frac{2 \pi \tilde{y}_{i}}{(Q \Delta \tilde{y})}
\end{array}\right.
$$

being the $3 \mathrm{D}$ spatial frequencies. In Algorithm 1, we summarize the proposed procedures of OFDM-MIMO radar echo preprocessing.

\section{OFDM-MIMO Radar High-Resolution Imaging via MD-UESPRIT}

In this section, we use the Multidimensional Unitary-ESPRIT (MD-UESPRIT) algorithm to simultaneously estimate the $3 \mathrm{D}$ spatial frequencies $\left\{\omega_{d, i} \mid d=1,2,3, i=1, \ldots, I\right\}$ with the spatial spectrum echo $\left\{s_{n, p, q}\right\}$ and further reconstruct the scatterers. Since $\left\{s_{n, p, q}\right\}$ is only one snapshot available, we first employ a Multidimensional Spatial Smoothing (MD-SS) technique to restore the full-rank echo matrix. Including the backward-forward averaging, the unitary transformation then takes advantage of the complex conjugate of the initial data matrix and doubles the available snapshots. Finally, the $3 \mathrm{D}$ spatial frequencies are automatically paired, calculating the joint eigenvalue decomposition (JEVD) of the estimated matrix pencils.

3.1. Multidimensional Spatial Smoothing. MD-SS is introduced in this section to restore the full-rank matrix. Let $D \in \mathbb{Z}^{+}$as the number of dimensions, stack the data $\left\{s_{l_{1}, \ldots, l_{D}} \mid d=1, \ldots, D, l_{d}=0, \ldots, L_{d}-1\right\}$ into an $L_{1} \times \cdots \times$ $L_{D}$ array, and scan it by an $R_{1} \times \cdots \times R_{D}$ window. The sliding step is 1 ; hence, there are $M=\prod_{d=1}^{D} M_{d}$ windows altogether, with $M_{d}=L_{d}-R_{d}+1$. Set the scanning permutation as $(1, \ldots, D)$ and denote $\left[m_{1}, \ldots, m_{D}\right]\left(m_{d} \in\left[0, M_{d}-1\right]\right)$ as the initial index of the $\left(m=m_{D} \prod_{d=1}^{D-1} M_{d}+\right.$ $\left.m_{D-1} \prod_{d=1}^{D-2} M_{d}+\cdots+m_{1}\right)$-th $\quad$ window; $\quad$ an $\quad\left(R=\prod_{d=1}^{D}\right.$ $R_{d}$ )-length vector $\mathbf{s}_{m}$ is then formed with the elements in the window, which is organized by

$$
\mathbf{s}_{m}=\left[s_{m_{1}, \ldots, m_{D}}, \ldots, s_{m_{1}+R_{1}-1, \ldots, m_{3}}, s_{m_{1}, m_{2}+1, \ldots, m_{D}}, \ldots, s_{m_{1}, m_{2}+R_{2}-1, \ldots, m_{D}}, \ldots, s_{m_{1}+R_{1}-1, \ldots, m_{D}+R_{D}-1}\right]^{\mathrm{T}}=\mathbf{A} \boldsymbol{\sigma}_{m}+n_{m}
$$

where $\mathbf{A}=\mathbf{A}_{D} \oplus \cdots \oplus \mathbf{A}_{1} \in \mathbb{C}^{R \times I}$ is the final steering matrix with $R=\prod_{d=1}^{D} R_{d}$ and

$$
A_{d}=\left[\begin{array}{ccc}
1 & \cdots & 1 \\
e^{j \omega_{d, 1}} & \cdots & e^{j \omega_{d, I}} \\
\vdots & \ddots & \vdots \\
e^{j\left(R_{d}-1\right) \omega_{d, 1}} & \cdots & e^{j\left(R_{d}-1\right) \omega_{d, I}}
\end{array}\right] \in \mathbb{C}^{R_{d} \times I}
$$

is the steering matrix for the $d$-th dimension. $\sigma_{m}=\left[\widetilde{\sigma}_{1, m}, \ldots, \widetilde{\sigma}_{i, m}, \ldots, \widetilde{\sigma}_{I, m}\right]^{\mathrm{T}}$ is the $m$-th signal vector with $\tilde{\sigma}_{i, m}=\tilde{\sigma}_{i} \exp \left\{j \sum_{d=1}^{D} m_{d} \omega_{d, i}\right\}$, and $\mathbf{n}_{m}$ is the noise vector within the $m$-th sliding window. The final matrix after spatial smoothing could be expressed as

$$
\mathbf{S}=\mathbf{A} \sum+\mathbf{N} \in \mathbb{C}^{R \times M}
$$

with $\mathbf{S}=\left[\mathbf{s}_{0}, \ldots, \mathbf{s}_{M-1}\right], \quad \sum=\left[\sigma_{0}, \ldots, \sigma_{M-1}\right] \in \mathbb{C}^{I \times M}$, and $\mathbf{N}=\left[\mathbf{n}_{0}, \ldots, \mathbf{n}_{M-1}\right] \in \mathbb{C}^{R \times M}$. Specifically, in this $3 \mathrm{D}$ imaging application for OFDM-MIMO radar, set $D=3, L_{1}=N$, $L_{2}=P$, and $L_{3}=Q$.

3.2. Unitary Transformation via Backward-Forward Averaging. Taking advantage of the complex conjugate of the data matrix, define the extended data matrix including the backward-forward averaging as

$$
\widetilde{\mathbf{S}}=\left[\mathbf{S}, \prod_{R} \mathbf{S}^{*} \prod_{M}\right] \in \mathbb{C}^{R \times 2 M},
$$

which doubles the available number of snapshots, with $\prod_{m}=\left[{ }_{1} \cdot\right]_{m \times m}$. It could be deduced that $\widetilde{\mathbf{S}}$ is centro-

Hermitian that 


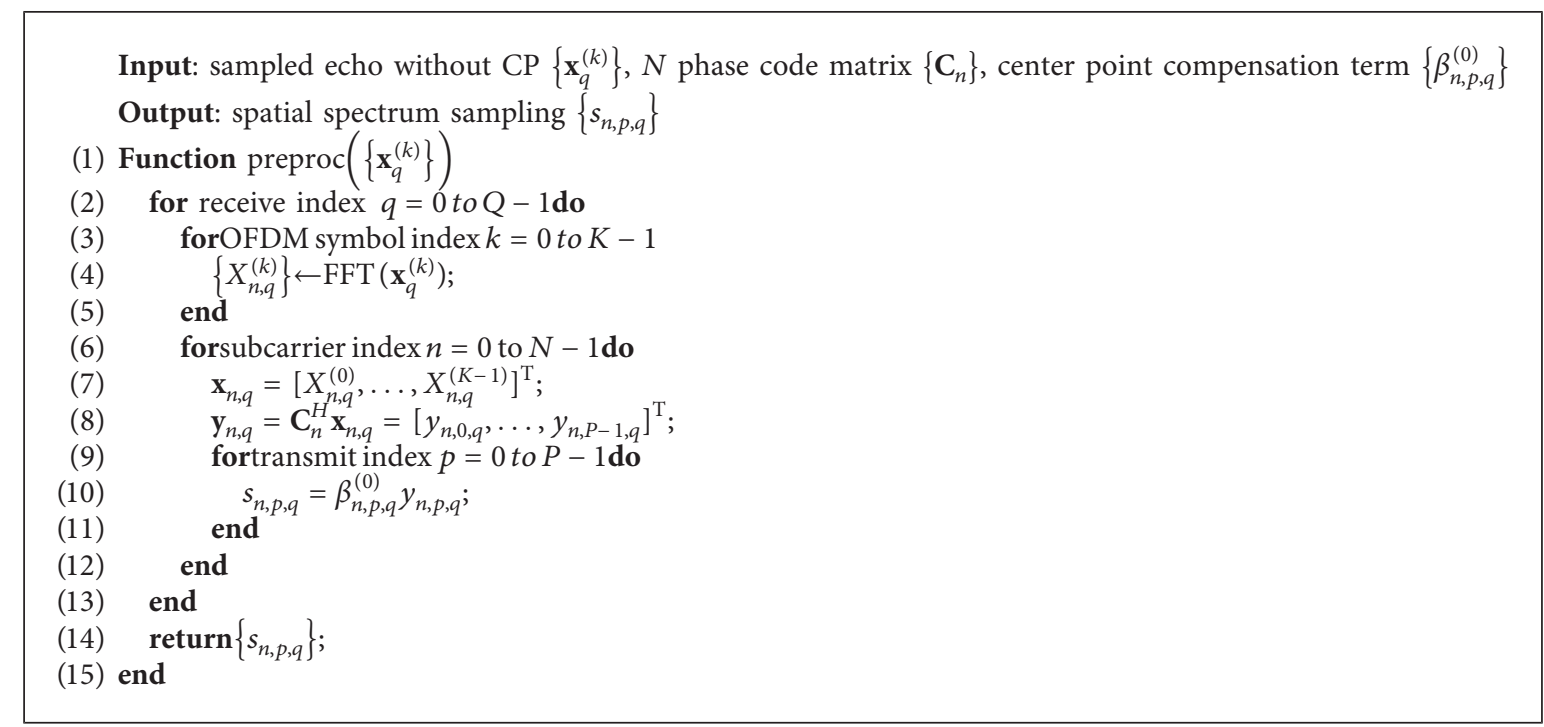

Algorithm 1: OFDM-MIMO radar echo preprocessing.

$$
\prod_{R} \widetilde{\mathbf{S}}^{*} \prod_{2 M}=\widetilde{\mathbf{S}}
$$

According to the theorem in [39], with the unitary matrix

$$
\mathbf{Q}_{2 m+1}=\frac{1}{\sqrt{2}}\left[\begin{array}{ccc}
\mathbf{I}_{m} & \mathbf{0} & j \mathbf{I}_{m} \\
\mathbf{0}^{\mathrm{T}} & \sqrt{2} & \mathbf{0}^{\mathrm{T}} \\
\boldsymbol{\Pi}_{m} & \mathbf{0} & -j \boldsymbol{\Pi}_{m}
\end{array}\right]
$$

(the center column and row vector are dropped for even case), the following matrix

$$
\mathbf{Z}=\mathscr{T}(\mathbf{S})=\mathbf{Q}_{R}^{H} \widetilde{\mathbf{S}} \mathbf{Q}_{2 M} \in \mathbb{R}^{R \times 2 M}
$$

is real-valued. To simplify the computation, set $\mathbf{S}=\left[\begin{array}{lll}\mathbf{S}_{1}^{\mathrm{T}} & \mathbf{s} & \mathbf{S}_{2}^{\mathrm{T}}\end{array}\right]^{\mathrm{T}}$, where submatrices $\mathbf{S}_{1}$ and $\mathbf{S}_{2}$ are of same size (the row vector $\mathbf{s}^{\mathrm{T}}$ is dropped when $R$ is even); the realvalued matrix $\mathbf{Z}$ in (26) could be straightforwardly calculated as

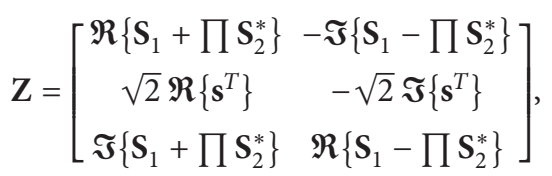

and only $R \times 2 M$ real additions are needed. Estimate the scatterer number $I$ [52-54] (the specific procedures of which are ignored in this paper), and the signal subspace $\mathbf{E}_{s}$ consists of the $I$ dominant left singular vectors of $\mathbf{Z}$. Correspondingly, the $I$ dominant left singular vectors of $\mathbf{S}$ are $\mathbf{Q}_{R} \mathbf{E}_{s}$ based on the corollary in [39].

3.3. Matrix Pencils Construction. D matrix pencils are constructed in this section exploiting the shift-invariance property of the uniform linear array manifold $\mathbf{A}_{d}$ in (20). For the $d$-th $(d=1, \ldots, D)$ steering matrix, we have

$$
\mathbf{J}_{d, 1}^{(0)} \mathbf{A}_{d} \boldsymbol{\Phi}_{d}^{(0)}=\mathbf{J}_{d, 2}^{(0)} \mathbf{A}_{d}
$$

where the selection matrices are $\mathbf{J}_{d, 1}^{(0)}=\left[\begin{array}{ll}\mathbf{I}_{R_{d}-1} & \mathbf{0}_{\left(R_{d}-1\right) \times 1}\end{array}\right]$, $\mathbf{J}_{d, 2}^{(0)}=\left[\begin{array}{ll}\mathbf{0}_{\left(R_{d}-1\right) \times 1} & \mathbf{I}_{R_{d}-1}\end{array}\right]=\prod_{R_{d}-1} \mathbf{J}_{d, 1}^{(0)} \prod_{R_{d}}$, and $\Phi_{d}^{(0)}=\operatorname{diag}$ $\left\{\exp \left(j \omega_{d, 1}\right), \ldots, \exp \left(j \omega_{d, I}\right)\right\}$ which is a diagonal matrix.

Given the selection matrices

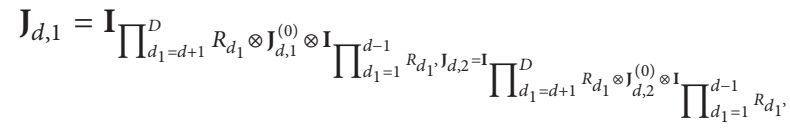

note that $\mathbf{I}_{\prod_{d_{1}=d+1}^{D} R_{d_{1}}}^{D}=1$ when $d=D$, and $\mathbf{I}_{\prod_{d_{1}=1}^{d-1} R_{d_{1}}}=1$ when $d=1$. Based on the Kronecker product properties $(A \otimes B)$ $(C \otimes D)=(A C) \otimes(B D), A \otimes(B \otimes C)=A \otimes B \otimes C, \quad$ the equations

$$
\begin{gathered}
\mathbf{J}_{d, 1} \mathbf{A} \Phi_{d}^{(0)}=\mathbf{J}_{d, 2} \mathbf{A} \\
\mathbf{J}_{d, 2}=\prod_{R-R_{d}} \mathbf{J}_{d, 1} \prod_{R}
\end{gathered}
$$

are established. Since $\mathbf{A}$ and $\left(\mathbf{Q}_{R} \mathbf{E}_{s}\right)$ span the same vector space, that is, there exists a nonsingular matrix $\mathbf{T}$ that $\mathbf{A}=\mathbf{Q}_{R} \mathbf{E}_{s} \mathbf{T}$, (30) could be rewritten as

$$
\mathbf{J}_{d, 1} \mathbf{Q}_{R} \mathbf{E}_{s} \mathbf{T} \Phi_{d}^{(0)}=\mathbf{J}_{d, 2} \mathbf{Q}_{R} \mathbf{E}_{s} \mathbf{T}
$$

Furthermore, based on (31),

$$
\mathbf{Q}_{R-R_{d}}^{H} \mathbf{J}_{d, 2} \mathbf{Q}_{R}=\mathbf{Q}_{R-R_{d}}^{H} \prod_{R-R_{d}} \mathbf{J}_{d, 1} \prod_{R} \mathbf{Q}_{R}=\left(\mathbf{Q}_{R-R_{d}}^{H} \mathbf{J}_{d, 1} \mathbf{Q}_{R}\right)^{*}
$$

Therefore, combining (32) and (33) yields

$$
\mathbf{K}_{d, 1} \mathbf{E}_{s} \Psi_{d}=\mathbf{K}_{d, 2} \mathbf{E}_{s},
$$

where

$$
\begin{aligned}
& \mathbf{K}_{d, 1}=\mathfrak{R}\left\{\mathbf{Q}_{R-R_{d}}^{H} \mathbf{J}_{d, 2} \mathbf{Q}_{R}\right\}, \\
& \mathbf{K}_{d, 2}=\mathfrak{\Im}\left\{\mathbf{Q}_{R-R_{d}}^{H} \mathbf{J}_{d, 2} \mathbf{Q}_{R}\right\},
\end{aligned}
$$


and $\quad \Psi_{d}=\mathbf{T} \Phi_{d} \mathbf{T}^{-1}$, with $\Phi_{d}=\operatorname{Diag}\left\{\tan \left(\omega_{d, 1} / 2\right), \ldots\right.$, $\left.\tan \left(\omega_{d, I} / 2\right)\right\}$. Basically, the $d$-th pencil $\Psi_{d}$ could be estimated via least square (LS) technique as

$$
\widehat{\mathbf{\Psi}}_{d}=\left(\mathbf{K}_{d, 1} \mathbf{E}_{s}\right)^{\dagger}\left(\mathbf{K}_{d, 2} \mathbf{E}_{s}\right) \text {. }
$$

3.4. Location Estimation via JEVD. In [42], the matrix $\mathbf{T}$ is estimated by computing the EVD of the matrix $\widehat{\Psi}=\sum_{d=1}^{D} \alpha_{d} \widehat{\Psi}_{d}$, where $\alpha_{d}$ is the coefficient satisfying $\sum_{d=1}^{D} \alpha_{d}=1, \alpha_{d}>0$. However, the diagonalizing performance relies heavily on the choice of coefficients $\left\{\alpha_{d}\right\}$, which are difficult to choose; meanwhile, the method has poor robustness with perturbations. In our work, the joint eigenvalue decomposition (JEVD) technique is adopted to jointly diagonalize the $D$ matrix pencils, as the following way:

$$
\widehat{\Psi}_{d}=\widehat{\mathbf{T}} \widehat{\Phi}_{d} \widehat{\mathbf{T}}^{-1}
$$

where $\widehat{\Psi}_{d}, \widehat{\Phi}_{d}$, and $\widehat{\mathbf{T}}$ are real. The uniqueness condition of the JEVD problem [55] is as follows:

Construct the matrix

$$
\boldsymbol{\Omega}=\left[\begin{array}{ccc}
\phi_{1}^{(1)} & \cdots & \phi_{1}^{(D)} \\
\vdots & \ddots & \vdots \\
\phi_{I}^{(1)} & \cdots & \phi_{I}^{(D)}
\end{array}\right] \in \mathbb{C}^{I \times D},
$$

where $\phi_{i}^{(d)}$ is the $i$-th diagonal term in $\widehat{\Phi}_{d}$. JEVD is unique if and only if all the rows of $\Omega$ are distinct.

JEVD algorithms such as the "sh-rt" algorithm [47], JUST algorithm [48], JDTM algorithm [49], and JET algorithm [50] are Jacobi-like methods based on different matrix decomposition techniques. "sh-rt" has the poorest eigenvector estimation performance against noise [56]. JUST is slow to converge. JDTM requires only a few iterations before convergence but has high computation complexity per round. Conversely, JET decreases the numerical complexity per iteration but needs more iterations to converge. The
JEVD based on a Taylor Expansion (JDTE) for real matrices is proposed in [51], which is also iterative but has no sweeping steps like Jacobi approaches. JDTE decreases the overall computational cost by limiting both the average number of iterations and the cost per iteration. Here, we choose JDTE for the estimation of $\widehat{\mathbf{T}}$ and $\left\{\widehat{\Phi}_{d}\right\}$.

In JDTE, an iteration procedure is used for JEVD. Initializing a nonsingular matrix $\mathbf{H}_{0}$ yields

$$
\Phi_{d}^{(0)}=\mathbf{H}_{0}^{-1} \widehat{\Psi}_{d} \mathbf{H}_{0},
$$

whereas at each iteration, a matrix $\mathbf{H}_{m},(m=1,2, \ldots)$ is computed to update $\Phi_{d}^{(m-1)}$ as

$$
\boldsymbol{\Phi}_{d}^{(m)}=\mathbf{H}_{m}^{-1} \boldsymbol{\Phi}_{d}^{(m-1)} \mathbf{H}_{m} .
$$
built as

The diagonalization parameter corresponding to $\mathbf{H}_{m}$ is

$$
c\left(\mathbf{H}_{m}\right)=\sum_{d=1}^{D}\left\|\boldsymbol{\Phi}_{d}^{(m)}-\boldsymbol{\Lambda}_{d}^{(m)}\right\|_{2}^{2}=\sum_{d=1}^{D}\left\|\mathbf{O}_{d}^{(m)}\right\|_{2}^{2}
$$

where $\|\cdot\|_{2}$ is the Euclidean norm of a real-valued matrix, $\Lambda_{d}^{(m)}=\operatorname{Diag}\left\{\operatorname{diag}\left\{\Phi_{d}^{(m)}\right\}\right\}$ is a diagonal matrix consisting of the diagonal terms of $\Phi_{d}^{(m)}$, and $\mathbf{O}_{d}^{(m)}=\Phi_{d}^{(m)}-\Lambda_{d}^{(m)}$. Easily, if the algorithm achieves convergence after $M$ iterations, then the final estimations are $\widehat{\mathbf{T}}=\prod_{m=0}^{M} \mathbf{H}_{m}$ and $\widehat{\Phi}_{d}=\Phi_{d}^{(M)}$.

The index $m$ is afterwards dropped to simplify the notations. Rewrite the matrices $\mathbf{H}=\mathbf{I}+\mathbf{Z}, \Phi_{d}=\Lambda_{d}+\mathbf{O}_{d}$, and take them into (40) and (41). Suppose that we are close to the final solution; hence, $\|\mathbf{Z}\|,\left\|\mathbf{O}_{d}\right\| \ll 1$, the first order Taylor approximation of (40), gives

$$
\begin{aligned}
(\mathbf{I}-\mathbf{Z})^{-1} \boldsymbol{\Phi}_{d}(\mathbf{I}+\mathbf{Z}) & \approx(\mathbf{I}-\mathbf{Z}) \boldsymbol{\Phi}_{d}(\mathbf{I}+\mathbf{Z}) \approx \boldsymbol{\Phi}_{d}-\mathbf{Z} \boldsymbol{\Phi}_{d}+\boldsymbol{\Phi}_{d} \mathbf{Z} \\
& \approx \Lambda_{d}+\mathbf{O}_{d}-\mathbf{Z} \Lambda_{d}+\Lambda_{d} \mathbf{Z},
\end{aligned}
$$

which is taken into (41) that the diagonalization parameter $c$ towards $\mathbf{Z}$ is

$$
c(\mathbf{Z})=\sum_{d=1}^{D}\left\|\mathbf{O}_{d}-\mathbf{Z} \boldsymbol{\Lambda}_{d}+\boldsymbol{\Lambda}_{d} \mathbf{Z}\right\|^{2}=\sum_{i=1}^{I} \sum_{\substack{j=1 \\ j \neq}}^{I-1} \sum_{d=1}^{D}\left(o_{i, j}^{(d)}+\left(\lambda_{i, i}^{(d)}-\lambda_{j, j}^{(d)}\right) z_{i, j}\right)^{2} \doteq \sum_{i=1}^{I} \sum_{\substack{j=1 \\ j \neq i}}^{I} f\left(z_{i, j}\right),
$$

where $o_{i, j}^{(d)}, \lambda_{i, i}^{(d)}$, and $z_{i, j}$ are the corresponding element of matrices $\mathbf{O}_{d}, \Lambda_{d}$, and $\mathbf{Z}$, respectively, and $f\left(z_{i, j}\right)=\sum_{d=1}^{D}\left(o_{i, j}^{(d)}+\left(\lambda_{i, i}^{(d)}-\lambda_{j, j}^{(d)}\right) z_{i, j}\right)^{2}$.

Minimizing $c(\mathbf{Z})$ equals to minimizing each $f\left(z_{i, j}\right)$. Let

$$
\frac{\partial f\left(z_{i, j}\right)}{\partial z_{i, j}}=2 \sum_{d=1}^{D}\left(o_{i, j}^{(d)}+\left(\lambda_{i, i}^{(d)}-\lambda_{j, j}^{(d)}\right) z_{i, j}\right)\left(\lambda_{i, i}^{(d)}-\lambda_{j, j}^{(d)}\right)=0,
$$

and consequently, the analytical solution of $z_{i, j}$ is

$$
z_{i, j}=-\frac{\sum_{d=1}^{D} o_{i, j}^{(d)}\left(\lambda_{i, i}^{(d)}-\lambda_{j, j}^{(d)}\right)}{\sum_{d=1}^{D}\left(\lambda_{i, i}^{(d)}-\Lambda_{j, j}^{(d)}\right)^{2}}
$$

The algorithm is stopped if the iteration number $m$ reaches the maximum $M_{\max }$ or the following condition is fulfilled: 


$$
\Delta c=\frac{\left|c\left(\mathbf{H}_{m}\right)-c\left(\mathbf{H}_{m-1}\right)\right|}{c\left(\mathbf{H}_{m-1}\right)} \leq \varepsilon
$$

The performance relies significantly on the choice of initial input $\mathbf{H}_{0}$. Here, we choose

$$
\mathbf{H}_{0}=\min _{\operatorname{evd}\left\{\widehat{\Psi}_{d}\right\}}\left\{c\left(\operatorname{evd}\left\{\widehat{\Psi}_{d}\right\}\right)\right\},
$$

where $\operatorname{evd}\{\cdot\}$ returns the matrix of eigenvectors and $\min \{\cdot\}$ denotes the minimum.

With the final estimation $\widehat{\Phi}_{d}$, extracting the diagonal elements yields the estimation of the spatial frequencies,

$$
\widehat{\omega}_{d, i}=2 \tan ^{-1}\left(\widehat{\phi}_{i, i}^{(d)}\right),
$$

where $\widehat{\phi}_{i, i}^{(d)}$ is the $i$-th diagonal element in $\widehat{\Phi}_{d}$. So far, the spatial frequencies are estimated and automatically paired via the MD-UESPRIT algorithm.

The scatterers' locations in the radar coordinate system are then reconstructed with the estimated spatial frequencies based on (16) and (19), whereas the scattering intensities are estimated through the LS algorithm [39]. Procedures of the MD unitary-ESPRIT algorithm are summarized in Algorithm 2, where $\operatorname{svd}(\cdot, I)$ returns the dominant $I$ left singular vectors and JDTE $(\cdot)$ is JEVD via JDTE algorithm, which is listed in Algorithm 3.

3.5. Computational Complexity Analysis. We list the real multiplications required for each step in the imaging procedures in Table 1. For the OFDM-MIMO echo preprocessing, the subcarriers separation via FFT needs $4 \times O[Q K N \ln N]$ flops, where $N, K$, and $Q$ signify the number of the sampling points within one OFDM bit, the OFDM bits in one pulse, and the receive antennas, respectively; the decoding and center point compensation need $4 \times O[\operatorname{NPQ}(K+1)]$ flops, with $P$ denoting the transmit antenna number. For the imaging algorithm, the spatial smoothing and unitary transformation in (27) need no real multiplications, whereas the signal subspace and $3 \mathrm{D}$ spatial frequencies estimation involve only real-value computations after unitary transformation. Denote $L$ as the iteration number of JDTE algorithm, and the total numerical complexities are shown in Table 1.

\section{Simulation Results}

4.1. Imaging Results Comparison. In this section, we use the simulated data for the 127-point scatterer model of a Boeing 777 airplane to verify the performance of the proposed 3D imaging method. The target scatterer model is depicted in Figure 2, including the projection in each plane and the $3 \mathrm{D}$ image. For ease of presentation and comparison, the scattering amplitudes of the scatterers are set uniformly distributed, with the dynamical range being $10 \mathrm{~dB}$. The center $\mathbf{o}$ of the imaging area is $\mathbf{o}=[1767.8,3061.9,3535.5]^{\mathrm{T}} \mathrm{m}$, with the initial radial range $d_{0}=6 \mathrm{~km}$.

The MIMO radar system consists of $P=8$-transmit ULA with element spacing $d_{t}=4 \mathrm{~m}$ and $Q=16$-receive ULA with element spacing $d_{r}=2 \mathrm{~m}$. Each transmit antenna radiates the OFDM waveform isotropically in space. The transmitted OFDM waveform consists of $N=128$ subcarriers, the frequency interval $\Delta f=1 \mathrm{MHz}$, the OFDM bit/CP/symbol durations are $t_{b}=1 \mu \mathrm{s}, t_{c}=0.5 \mu \mathrm{s}$, and $t_{s}=1.5 \mu \mathrm{s}$, respectively, and $K=16$; hence, the bandwidth is $B w=N \Delta f=$ $128 \mathrm{MHz}$ and the pulse width is $t_{p}=K t_{s}=24 \mu \mathrm{s}$. After preprocessing, the size of the spatial spectrum data array is $128 \times 8 \times 16$.

Figure 3 compares the imaging results obtained, respectively, from the 3D-FFT, MD-MUSIC, and MD-ESPRIT in [42] and the proposed MD-UESPRIT algorithm. Each image is normalized by the values of the strongest points. All the tests are conducted with $\mathrm{SNR}=15 \mathrm{~dB}$, where SNR is defined as

$$
\mathrm{SNR}=20 \lg \frac{\sum_{q=0}^{\mathrm{Q}-1} E_{s, q}}{\sum_{q=0}^{\mathrm{Q}-1} E_{n, q}},
$$

with $E_{s, q}$ and $E_{n, q}$ being the signal/noise power, respectively, at the $q$-th receiver.

The $3 \mathrm{D}$-FFT result is computed with the zero-padded data array of size $256 \times 64 \times 128$. In Figure $3(a)$, the first three subfigures show the superimposed $2 \mathrm{D}$ images in $u-\tilde{x}$, $u-\tilde{y}$, and $\tilde{x}-\tilde{y}$ planes, respectively. The grid widths of the radial range and $2 \mathrm{D}$ cross ranges are $u_{0}=0.5859 \mathrm{~m}$, $\tilde{x}_{0}=0.7031 \mathrm{~m}$, and $\tilde{y}_{0}=0.7031 \mathrm{~m}$. The resolution of FFT spectrum is so low that we could hardly identify the target from the results. It can be seen in the last subfigure that the target could hardly be identified from the reconstructed image.

Set the smoothing window sizes as $(64,4,8)$; the snapshots after MD-SS are $M=\prod_{d=1}^{D} M_{d}=2925$. The MDMUSIC algorithm is adopted in Figure 3(b) with the same searching steps as in Figure 3(a). Mainlobe width and sidelobe level decrease significantly in the MUSIC spectrum compared with the FFT spectrum. In the last subfigure, peaks in the MUSIC spectrum are selected for target reconstruction, whereas the scattering amplitudes are not directly equal to the spectrum value but are also calculated via the LS algorithm. However, the reconstruction error is still high; meanwhile, it consumes a long time for $3 \mathrm{D}$ searching.

As can be seen in Figures 3(c) and 3(d), the imaging results of the ESPRIT-class methods are the specific locations and intensities of distinct scatterers without any sidelobes. The proposed method in Figure 3(d) has best imaging quality with high reconstruction accuracy and takes less time than MD-MUSIC and MD-ESPRIT.

The performance of the proposed algorithm is further demonstrated with 2D EM simulated data of an Airbus A320 airplane. The transmitted OFDM waveform consists of $N=$ 256 subcarriers with frequency interval $\Delta f=1 \mathrm{MHz}$; the bandwidth is hence $B w=256 \mathrm{MHz}$ with a carrier frequency of $f_{0}=10 \mathrm{GHz}$. 64-element ULA is adopted for receiving; the incident angle for each receiver is increased by $0.009^{\circ}$. The spatial spectrum echo is $256 \times 64$ after preprocessing. Set the initial incident/azimuth angle as $\left(90^{\circ}, 0^{\circ}\right)$ and $\left(90^{\circ}\right.$, $\left.45^{\circ}\right)$; the normalized imaging results are depicted in Figures 4(a) and 4(b), respectively. In each subfigure, the 
Input: $D$-dimension spatial spectrum samplings $\left\{s_{l_{1}, \ldots, l_{D}} \mid d=1, \ldots, D, l_{d}=0, \ldots L_{d}-1\right\}$, window widths $\left\{R_{d}\right\}$, selection matrix

$\left\{\mathbf{K}_{d, 1}\right\}$ and $\left\{\mathbf{K}_{d, 2}\right\}$, maximum iteration number $M_{\max }$, stopping threshold $\varepsilon$

Output: spatial frequencies $\left\{\left(\widehat{\omega}_{1, i}, \ldots, \widehat{\omega}_{D, i}\right)\right\}$

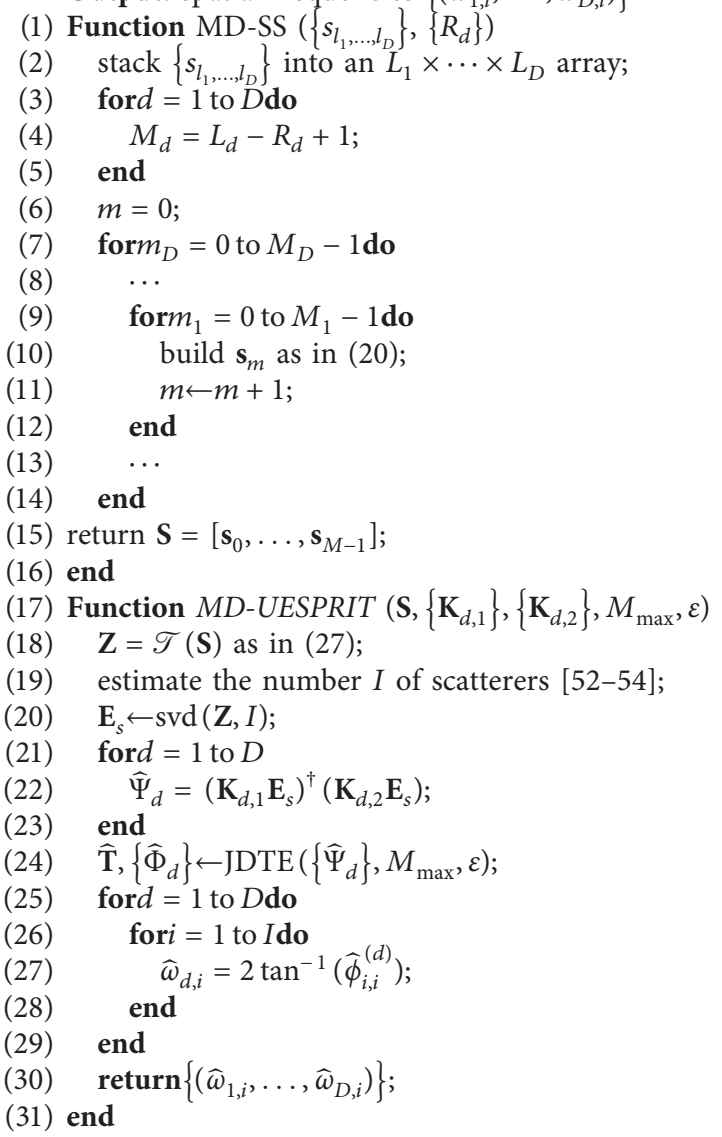

Algorithm 2: Imaging via the MD unitary-ESPRIT algorithm.

results of 2D-FFT, 2D-MUSIC, and 2D-ESPRIT are also shown as a comparison. Figure 4 indicates that the highresolution spatial frequency estimation methods such as MUSIC and ESPRIT provide better resolution images compared with the Fourier-based method. Among them, MUSIC is time-consuming with multidimensional searching. The proposed method has better reconstruction performance with fewer fake peaks and a dynamical range closer to that of the FFT method.
4.2. Reconstruction Accuracy Analysis. The reconstruction accuracy of 3D-UESPRIT estimation is analyzed in this section, corresponding to SNR, system parameters, and errors. As shown in Figure 5, we choose a target with fewer scatterers for ease of analysis with initial radial distance being $d_{0}=4 \mathrm{~km}$. The size of scatterers indicates the scattering amplitude. For each SNR and parameters combination, we carry out $M=200$ Monte Carlo simulations. The Root-Mean-Square Error (RMSE) is computed to measure the reconstruction precision and is expressed as

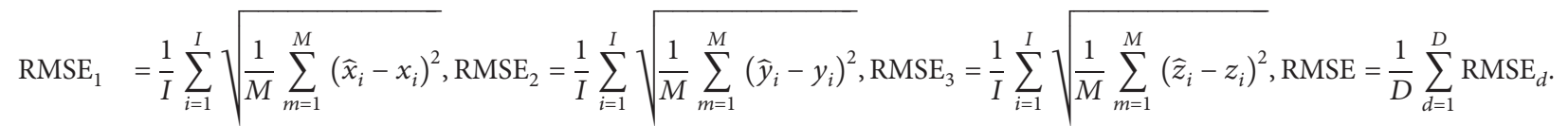

In Figure 6, SNR is varied from $-18 \mathrm{~dB}$ to $18 \mathrm{~dB}$ with $3 \mathrm{~dB}$ as the step. The four subfigures in Figure 6 show that RMSE decreases with the increase of SNR, especially when $\mathrm{SNR} \leq 0 \mathrm{~dB}$. Keeping the system parameters, the same with those in Figure 3(d), we utilize different window sizes for MD-SS in Figure 6(a). For each dimension, the window size decides the order of the final steering matrix and the incoherence property of multiple scatterers in matrix $\mathbf{S}$. 


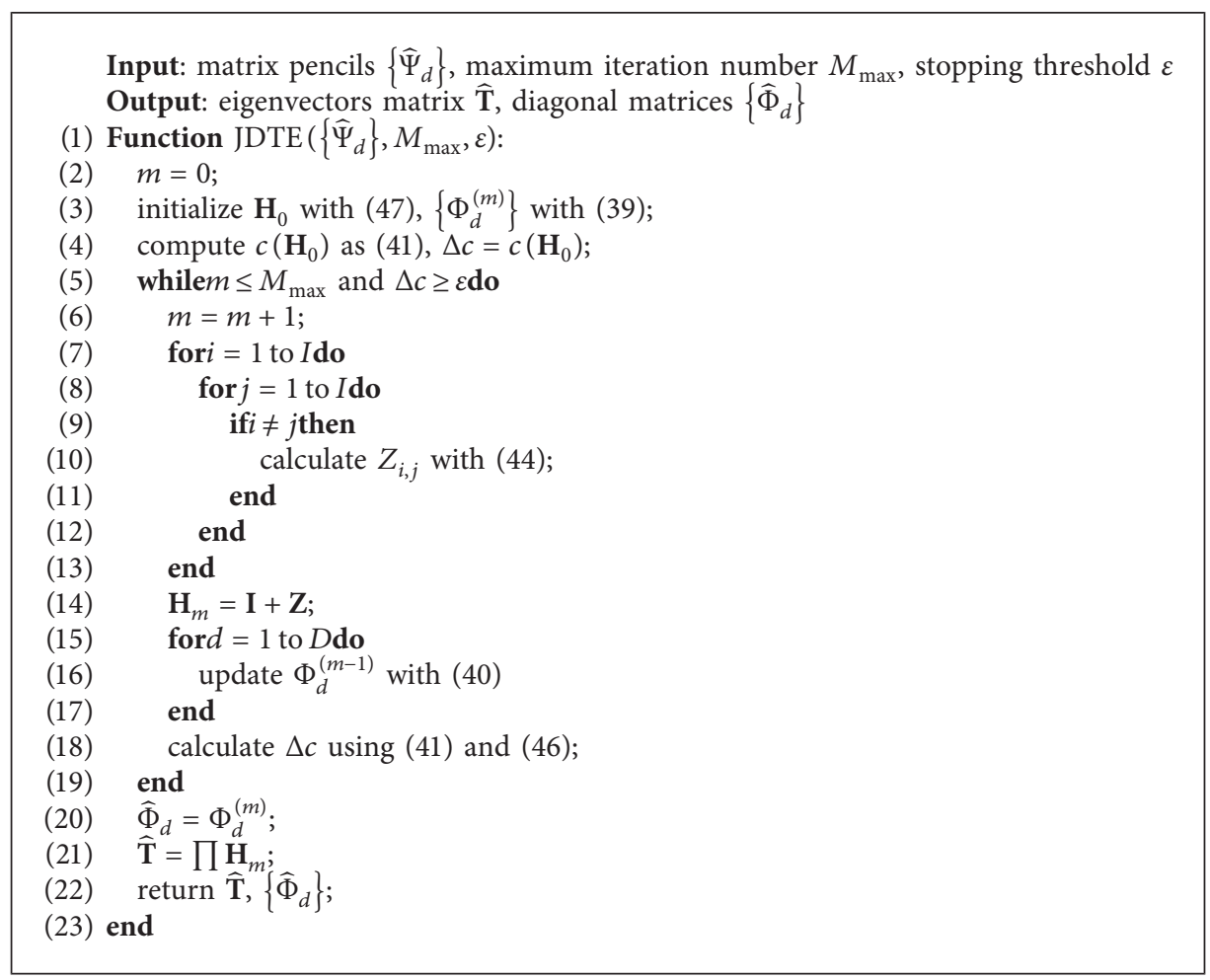

Algorithm 3: JEVD via JDTE.

TABLE 1: Computational complexity analysis.

\begin{tabular}{lc}
\hline Step & Real multiplications \\
\hline Preprocessing & $4 \times O[\mathrm{QKN} \ln N+\mathrm{QNP}(K+1)]$ \\
Spatial smoothing & - \\
Unitary transformation & - \\
SVD & $O\left[R^{3}\right]$ \\
Matrix pencils & $O\left[\left(3 R-\sum R_{d}\right)\left(2 R I+I^{2}\right)\right]$ \\
computation & $L \times O\left[I(I-1)(2 D+1)+2 I^{3}(D+1)\right]$ \\
\hline JEVD via JDTE
\end{tabular}

Figure 6(a) shows that, within a certain range, the estimation precision improves with the increase of the total matrix order $R=\prod_{d=1}^{D} R_{d}$, while after that, the estimation accuracy decreases on the contrary. This is because when $R$ exceeds some value, the coherence among scatterers increases that does not meet the criterion of the ESPRIT-class algorithm. Meanwhile, the identifiability of the algorithm [39] is defined as

$$
I_{\max }=\min \left\{\left(R_{1}-1\right) \prod_{d=2}^{D} R_{d}, R_{1}\left(R_{2}-1\right) \prod_{d=2}^{D} R_{d}, \ldots, \prod_{d=1}^{D-1}\left(R_{D}-1\right), 2 M\right\} .
$$

Therefore, considering both the estimation accuracy and the maximum estimable scatterer number, we choose $R_{d}=L_{d} / 2(d=1, \ldots, D)$ for the MD-UESPRIT algorithms.

Suppose other parameters be unchanged as in Figure 3(d); the subcarrier/transmit/receive array parameters are analyzed, respectively, in Figures 6(b) and 6(d). For each array, with the same element number, RMSE decreases with the increase of interelement spacings $\left(\Delta f, d_{t}, d_{r}\right)$, which is equivalent to the increase of bandwidth $B w$ and array lengths, while the maximum unambiguous intervals are

$$
u_{\max }=\frac{\alpha c}{(2 \Delta f)}, \quad \tilde{x}_{\max }=\frac{\lambda d_{0}}{d_{t}}, \tilde{y}_{\max }=\frac{\lambda d_{0}}{d_{r}},
$$

which are inversely proportional to the interelement spacings. Hence, the interelement spacings cannot increase without limitation, and the array lengths are subsequently restricted. With the bandwidth/array lengths unchanged, the estimation accuracy ameliorates with the increase of element numbers $(N, P, Q)$. However, both the system cost and computational complexity improve with more elements. Thus, we have to reach a compromise between the element number and interelement spacing when designing the system parameters.

Last but not least, reconstruction accuracy is analyzed concerning the system errors. Position errors in the transmit/receive antenna array are considered. The coordinate of $p$-th transmit antenna is set as $\mathbf{t}_{p}=\left[(p+\Delta p) d_{t}, 0,0\right]^{\mathrm{T}}$, where $\Delta p$ is a random value uniformly distributed in $\left[-\sigma_{d} / 2, \sigma_{d} / 2\right]$. Similarly, for $q$-th receive antenna, $r_{q}=\left[0,(q+\Delta q) d_{r}, 0\right]^{\mathrm{T}}$, and $\Delta q \sim \mathbb{U}\left(-\sigma_{d} / 2, \sigma_{d} / 2\right)$. For simplification, we also model the Doppler frequency compensation residual error on each subcarrier, a uniformly distributed random, i.e., the $n$-th subcarrier frequency after Doppler compensation is $f_{n}=f_{0}+(n+\Delta n) \Delta f$ and $\Delta n \sim \mathbb{U}\left(-\sigma_{f} / 2, \sigma_{f} / 2\right)$. RMSE curves with respect to $\sigma_{d}$ and $\sigma_{f}$ are depicted in Figure 7 , with $\mathrm{SNR}=5 \mathrm{~dB}$. For one thing, the estimation accuracy is sensitive to antenna position 

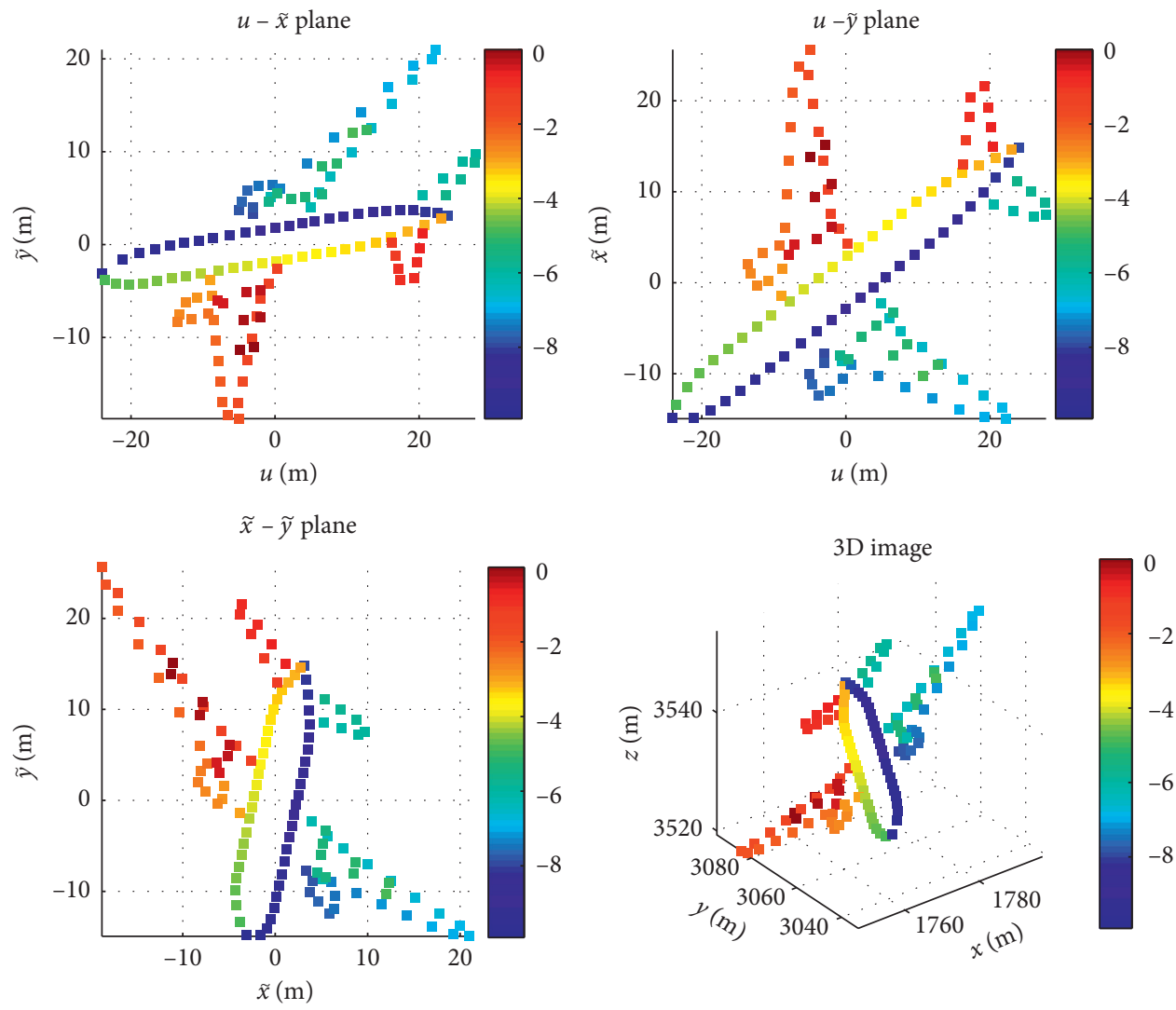

Figure 2: Scatterer model of a Boeing 777 plane.
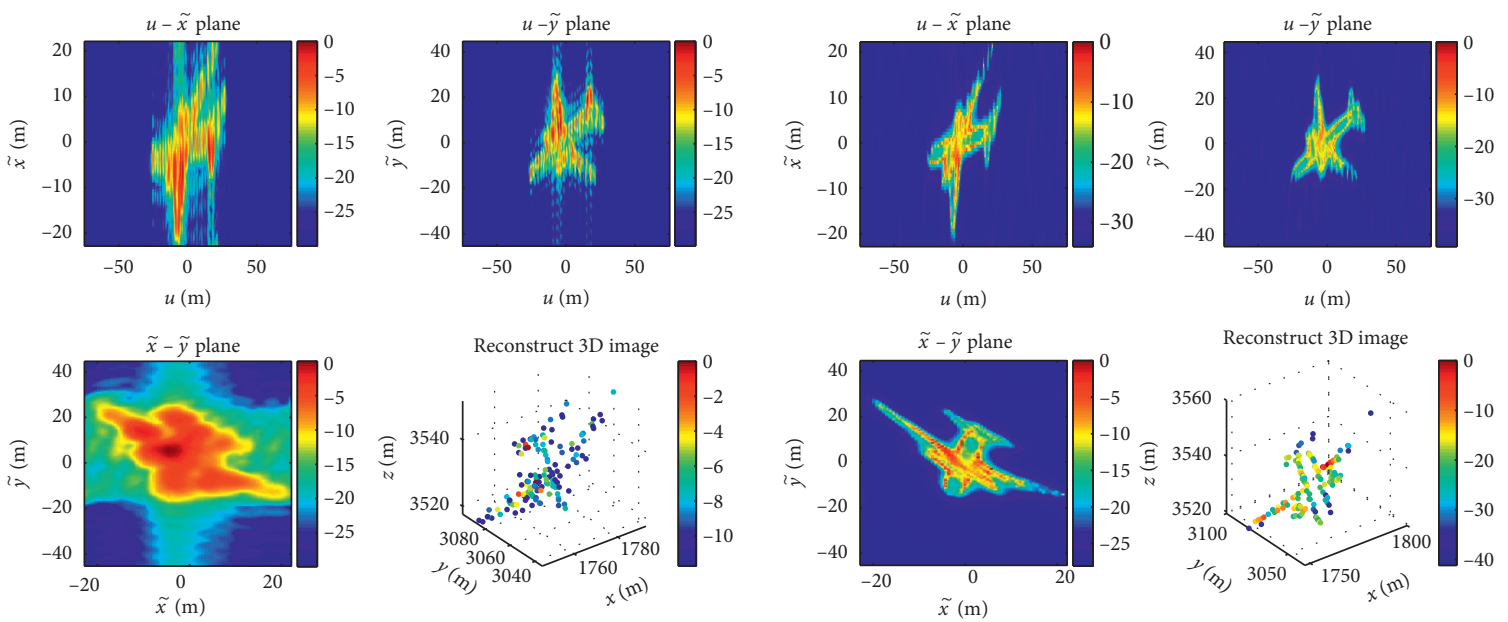

(a)

(b)

FIgURE 3: Continued. 

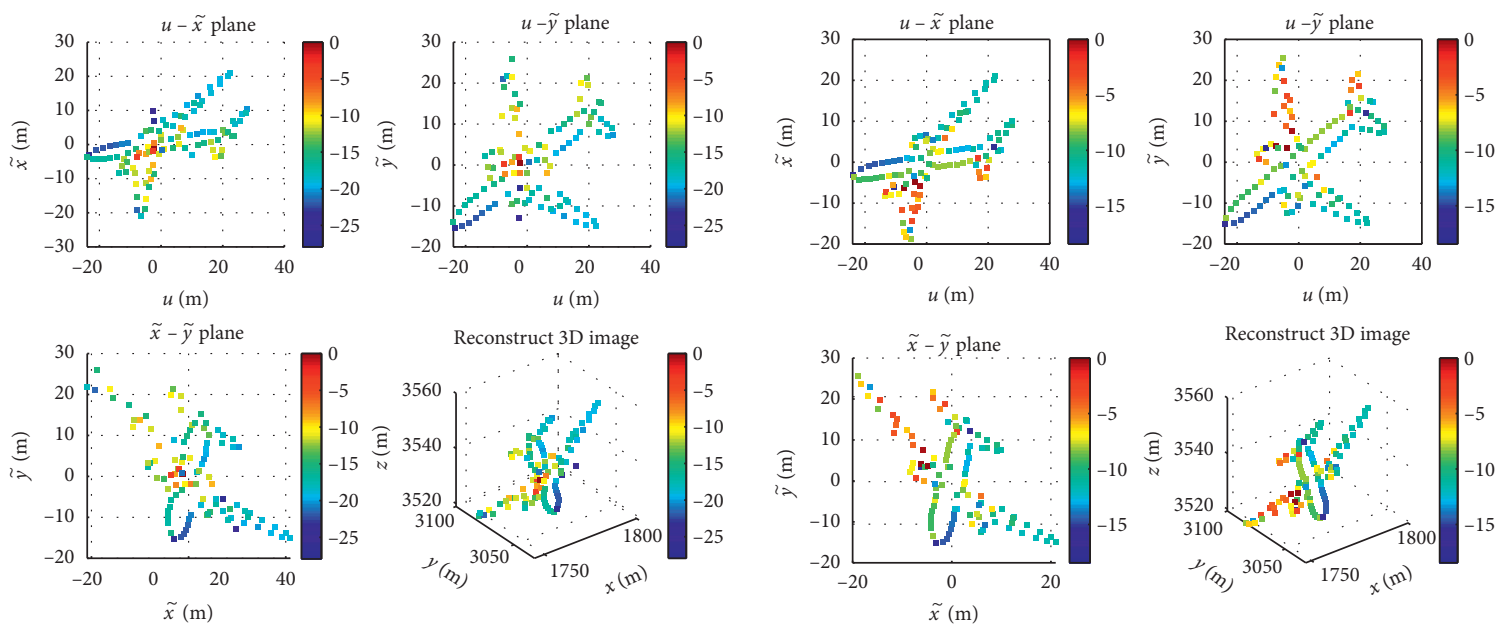

(c)

(d)

FIGURE 3: Imaging results of a Boeing 777 plane.
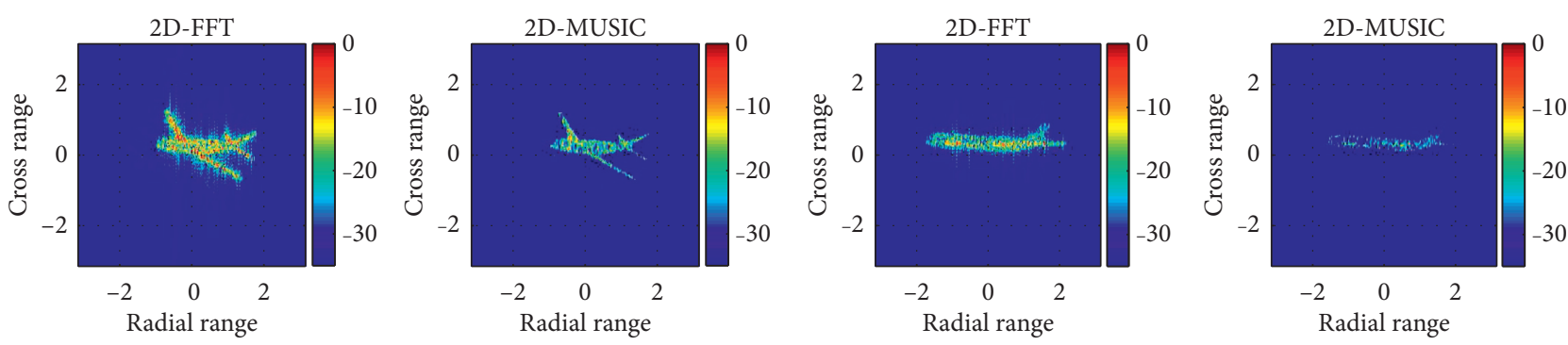

2D-ESPRIT

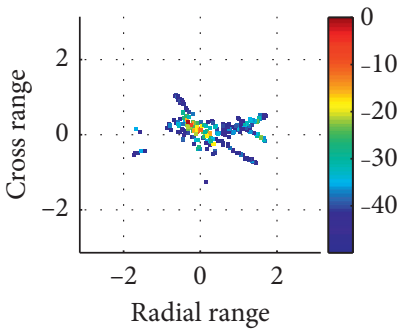

2D-UESPRIT

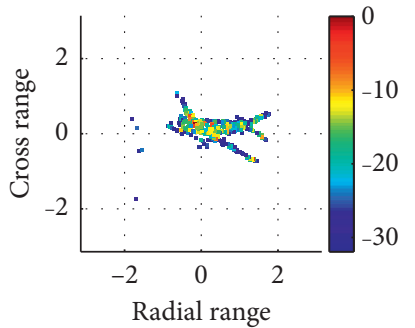

(a)
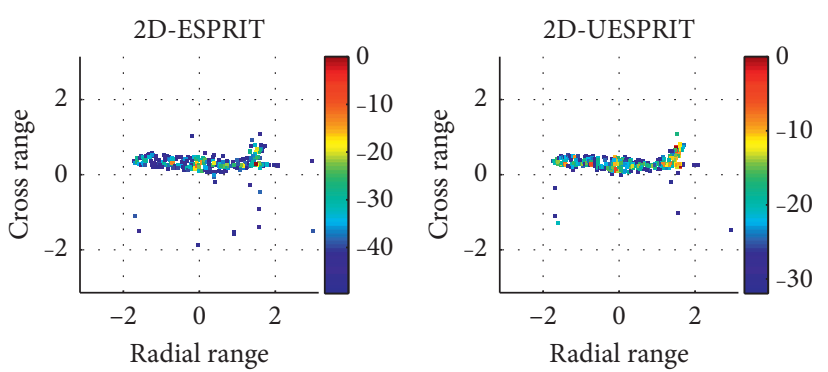

(b)

Figure 4: Imaging results of an Airbus A320 plane.

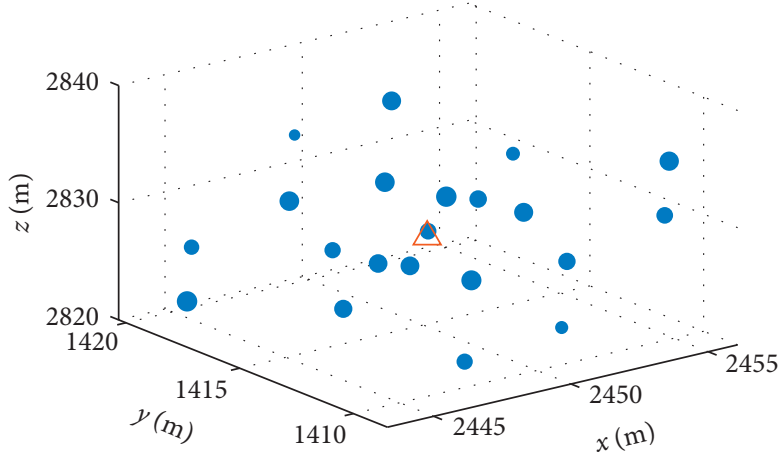

FiguRE 5: Simulated 3D target model with the red rectangular representing the imaging center. 


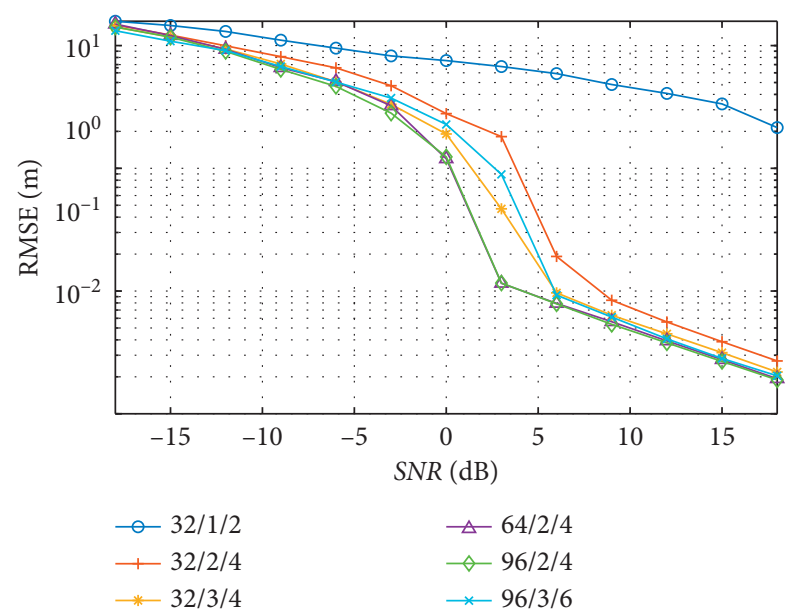

(a)

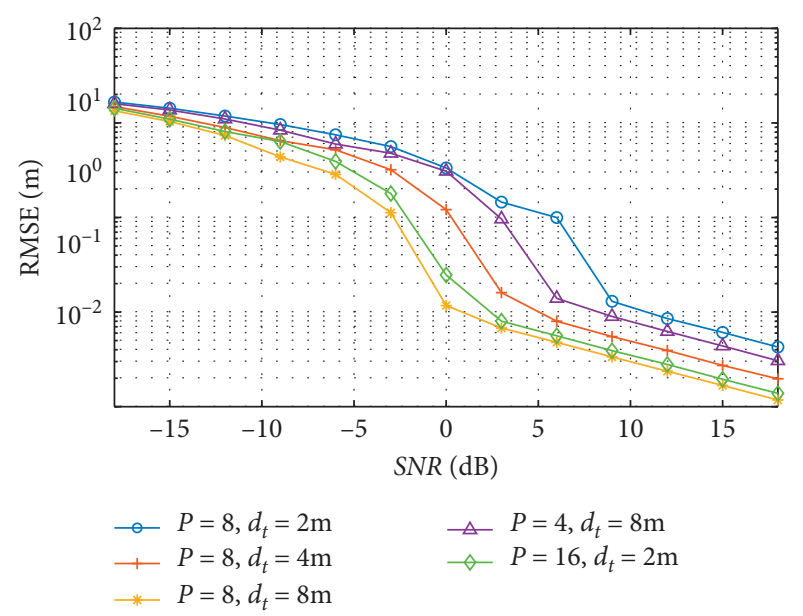

(c)

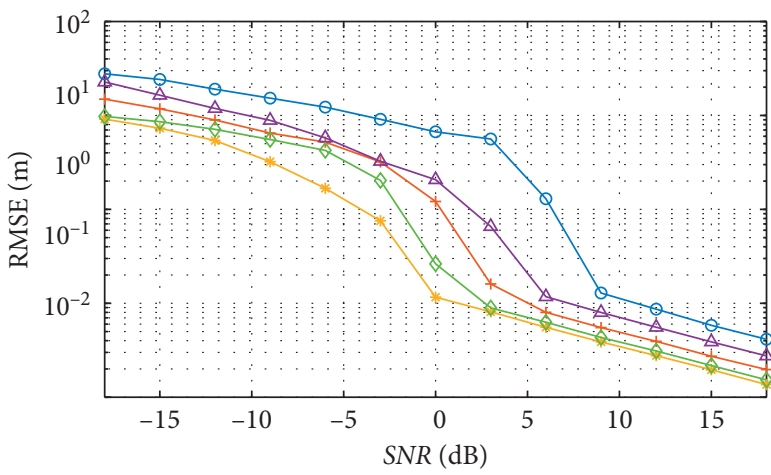

$\multimap N=128, \Delta f=0.5 \mathrm{MHz} \triangle N=64, \Delta f=2 \mathrm{MHz}$

$\rightarrow N=128, \Delta f=1 \mathrm{MHz} \rightarrow N=256, \Delta f=0.5 \mathrm{MHz}$

* $N=128, \Delta f=2 \mathrm{MHz}$

(b)

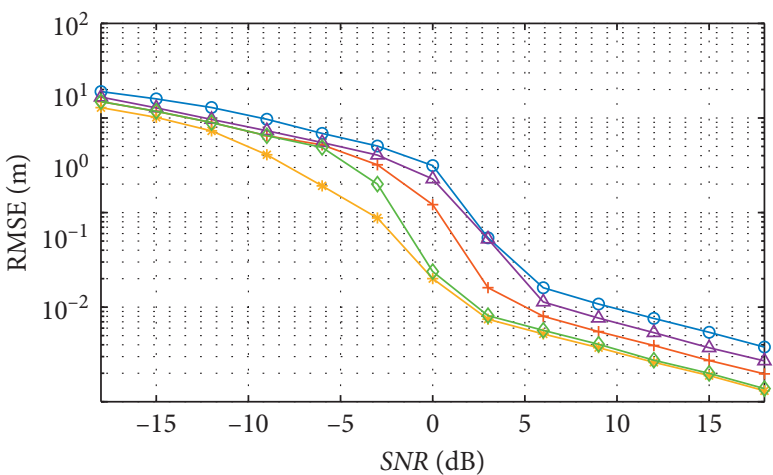

(d)

FIgURE 6: Reconstruction accuracy corresponding to SNR and parameters.

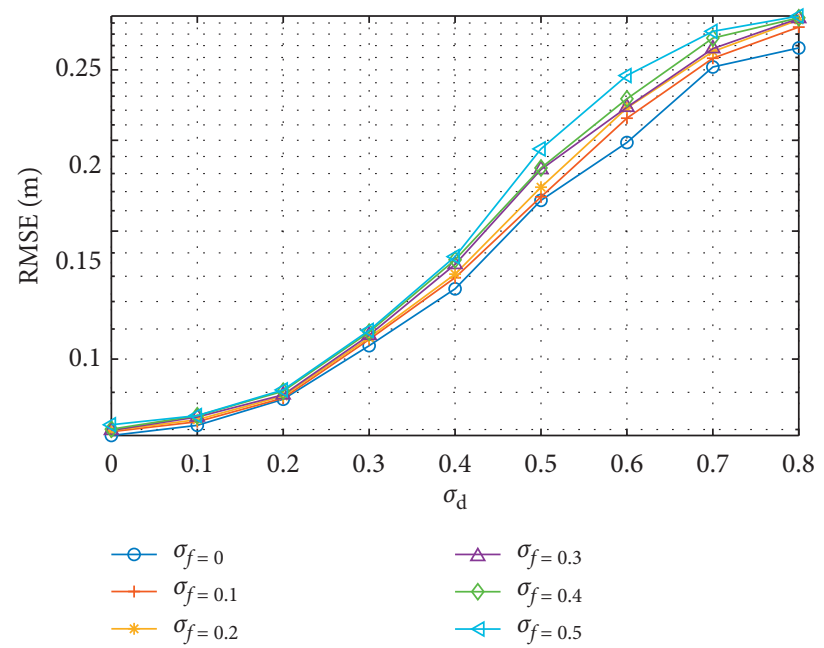

FIGURE 7: Reconstruction accuracy corresponding to antenna arrays and frequency errors. 
error; hence, effective antenna array calibration method is required before imaging. For another, with the system setting in the tests, the method has a tolerance of Doppler compensation error. However, we ignore the Intercarrier Inference (ICI) for subcarrier separation and decoding in our Doppler perturbation model. Therefore, more detailed considerations towards Doppler compensation error are required in the future work.

\section{Threats to Validity}

There are two threats to validity as follows.

The first threat is that we ignore the Doppler effect in our echo model by assuming the target velocity is zero or fully compensated. However, this condition is hard to achieve in reality. For one thing, we model the Doppler frequency shift in 4.2 as a random error in the subcarrier frequency array to measure the reconstruction accuracy relative to the Doppler effect. For another, the Doppler offset causes the interferences among subcarriers and transmitters [8], which is not considered in this paper.

The second threat is that the estimated signal subspace suffers from significant deviation with perturbations (including noise and array manifold error).The proposed method is not robust enough with SNR and system errors.

\section{Future Work}

As future work, we are planning to investigate some modifications of the proposed algorithm to make reconstruction more robust to SNR and system errors, which is significant for real applications. Secondly, we will build a complete signal model, including the Doppler effect, and propose an optimized transmitting scheme to mitigate the Doppler offset. Last but not least, we will build a physical experiment system to verify our method.

\section{Conclusion}

We present in this paper a high-resolution 3D imaging method for a MIMO-OFDM radar system with a single pulse. Orthogonal transmit and complete separation are achieved with OFDM structure and the preprocessing steps. We adopt the MD-UESPRIT algorithm to reconstruct the coordinates of scatterers simultaneously. The simulation results indicate that our proposed imaging algorithm has better reconstruction results compared with FFT, MD-MUSIC, and MD-ESPRIT. Parameters selection formulas about estimation accuracy, maximum unambiguous range, identifiability, and computational complexity are also provided in this paper.

\section{Data Availability}

The data used to support the findings of this study are included in the article.

\section{Conflicts of Interest}

The authors declare that there are no conflicts of interest regarding the publication of this paper.

\section{Acknowledgments}

This research was supported in part by the National Science Foundation of China under Contracts nos. 60872134, 61025006 , and 61921001.

\section{References}

[1] N. Levanon, "Multifrequency complementary phase-coded radar signal," IEE Proceedings-Radar, Sonar and Navigation, vol. 147 , no. 6 , p. $276,2000$.

[2] N. Levanon and E. Mozeson, "Multicarrier radar signal-pulse train and CW," IEEE Transactions on Aerospace and Electronic Systems, vol. 38, no. 2, pp. 707-720, 2002.

[3] E. Mozeson and N. Levanon, "Multicarrier radar signals with low peak-to-mean envelope power ratio," IEE Proceedings-Radar, Sonar and Navigation, vol. 150, no. 2, p. 71, 2003.

[4] C. Sturm, E. Pancera, T. Zwick, and W. Wiesbeck, "A novel approach to OFDM radar processing," in Proceedings of the IEEE Radar Conference, pp. 1-4, IEEE, Pasadena, CA, USA, May 2009.

[5] R. F. Tigrek, "A processing technique for OFDM-modulated wideband radar signals," Ph. D. thesis, Delft University of Technology, Delft, Netherlands, 2010.

[6] J. Zhao, K. Huo, and L. Xiang, "A chaos-based phase-coded OFDM signal for joint radar-communication systems," in Proceeding of the 12th International Conference on Signal Processing (ICSP), pp. 1997-2002, IEEE, Hangzhou, China, October 2014.

[7] J. Zhao, K. Huo, Y. Liu, and X. Yang, "Cyclic prefix based phase-coded OFDM radar doppler offset estimation and compensation," Journal of Electronics \& Information Technology, vol. 39, no. 4, pp. 938-944, 2017.

[8] F. Zhang, Z. Zhang, W. Yu, and T.-K. Truong, "Joint range and velocity estimation with intrapulse and intersubcarrier doppler effects for OFDM-based radcom systems," IEEE Transactions on Signal Processing, vol. 68, pp. 662-675, 2020.

[9] B. Donnet and I. Longstaff, "Combining MIMO radar with OFDM communications," in Proceedings of the European Radar Conference, pp. 37-40, IEEE, Manchester, UK, September 2006.

[10] C. Pfeffer, R. Feger, and A. Stelzer, "A stepped-carrier 77-GHz OFDM MIMO radar system with $4 \mathrm{GHz}$ bandwidth," in Proceedings of the 2015 European Radar Conference (EuRAD), pp. 97-100, IEEE, Paris, France, September 2015.

[11] Y. L. Sit, T. T. Nguyen, C. Sturm, and T. Zwick, "2D radar imaging with velocity estimation using a MIMO OFDMbased radar for automotive applications," in Proceedings of the European Radar Conference, pp. 145-148, IEEE, Berlin, Germany, September 2013.

[12] Y. L. Sit, T. T. Nguyen, and T. Zwick, "3D Radar imaging with a MIMO OFDM-based radar," in Proceedings of the AsiaPacific Microwave Conference Proceedings (APMC), pp. 6870, IEEE, Seoul, South Korea, November 2013.

[13] Z. Lin and Z. Wang, "Interleaved OFDM signals for MIMO radar," IEEE Sensors Journal, vol. 15, no. 11, pp. 6294-6305, 2015. 
[14] B. Nuss, Y. L. Sit, and T. Zwick, "3D radar image fusion using OFDM-based MIMO radar," in Proceedings of the German Microwave Conference (GeMiC), pp. 209-212, IEEE, Bochum, Germany, March 2016.

[15] J. Wang, L. Chen, and X. Liang, "Orthogonal waveform separation based on echo compression for airborne MIMO-SAR systems," The Journal of Engineering, vol. 2019, no. 19, pp. 6336-6340, 2019.

[16] J. Wang, X.-D. Liang, L.-Y. Chen, L.-N. Wang, and K. Li, "First demonstration of joint wireless communication and high-resolution SAR imaging using airborne MIMO radar system," IEEE Transactions on Geoscience and Remote Sensing, vol. 57, no. 9, pp. 6619-6632, 2019.

[17] H. Gor and B. Yang, "A novel OFDM-MIMO radar with nonequidistant dynamic subcarrier interleaving," in Proceedings of the European Radar Conference (EuRAD), pp. 45-48, London, UK, October 2016.

[18] G. Hakobyan, M. Ulrich, and B. Yang, "OFDM-MIMO radar with optimized nonequidistant subcarrier interleaving," IEEE Transactions on Aerospace and Electronic Systems, vol. 56, no. 1, pp. 572-584, 2020.

[19] Y.-He Cao, X.-G. Xia, and S.-H. Wang, "IRCI free Colocated MIMO radar based on sufficient cyclic prefix OFDM waveforms," IEEE Transactions on Aerospace and Electronic Systems, vol. 51, no. 3, 2015.

[20] X.-G. Xia, T. Zhang, and L. Kong, "MIMO OFDM radar IRCI free range reconstruction with sufficient cyclic prefix," IEEE Transactions on Aerospace and Electronic Systems, vol. 51, no. 3, pp. 2276-2293, 2015.

[21] V. Tarokh, H. Jafarkhani, and A. R. Calderbank, "Space-time block codes from orthogonal designs," IEEE Transactions on Information Theory, vol. 45, no. 5, pp. 1456-1467, 1999.

[22] K. Lu, S. Fu, and X.-G. Xia, "Closed-form designs of complex orthogonal space-time block codes of rates $<$ tex $>\$(k+1) /$ $(2 \mathrm{k}) \$</$ tex $>$ for $<$ tex $>\$ 2 \mathrm{k}-1 \$</$ tex $>$ or $<$ tex $>\$ 2 \mathrm{k} \$</$ tex $>$ transmit antennas," IEEE Transactions on Information Theory, vol. 51, no. 12, pp. 4340-4347, 2005.

[23] S. Das and B. Sundar Rajan, "Square complex orthogonal designs with no zero entry for any $2^{m}$ antennas," 2008, https:// arxiv.org/abs/0812.2458.

[24] G. Wang, X.-G. Xia, and V. C. Chen, "Three-dimensional ISAR imaging of maneuvering targets using three receivers," IEEE Transactions on Image Processing, vol. 10, no. 3, pp. 436-447, 2001.

[25] X. Xiaojian Xu and R. M. Narayanan, "Three-dimensional interferometric ISAR imaging for target scattering diagnosis and modeling," IEEE Transactions on Image Processing, vol. 10, no. 7, pp. 1094-1102, 2001.

[26] Q. Chen, M. Xing, L. Zhang, Z. Bao, and G. Xu, “Three-dimensional interferometric inverse synthetic aperture radar imaging with limited pulses by exploiting joint sparsity," IET Radar, Sonar \& Navigation, vol. 9, no. 6, pp. 692-701, 2015.

[27] X. Xie and Y. Zhang, "3D ISAR imaging based on MIMO radar array," in Proceedings of the 2nd Asian-Pacific Conference on Synthetic Aperture Radar, pp. 1018-1021, IEEE, Xian, China, October 2009.

[28] Y. Zhu, Y. Su, and W. Yu, "An ISAR imaging method based on MIMO technique," IEEE Transactions on Geoscience and Remote Sensing, vol. 48, no. 8, pp. 3290-3299, 2010.

[29] X. Hu, N. Tong, Y. Zhang, and Y. Wang, "3D imaging using narrowband MIMO radar and ISAR technique," in Proceedings of the International Conference on Wireless Communications \& Signal Processing (WCSP), pp. 1-5, IEEE, Nanjing, China, October 2015.
[30] G. Q. Duan, D. Wei, X. Y. Ma, and Y. Su, “Three-dimensional imaging via wideband MIMO radar system," IEEE Geoscience and Remote Sensing Letters, vol. 7, no. 3, pp. 445-449, 2010.

[31] C. Ma, T. S. Yeo, C. S. Tan, and Z. Liu, "Three-dimensional imaging of targets using colocated MIMO radar," IEEE Transactions on Geoscience and Remote Sensing, vol. 49, no. 8, pp. 3009-3021, 2011.

[32] J. Yang, W. Su, and H. Gu, "3D imaging using narrowband bistatic MIMO radar," Electronics Letters, vol. 50, no. 15, pp. 1090-1092, 2014.

[33] S. Ding, N. Tong, Y. Zhang, and X. Hu, "Super-resolution 3D imaging in MIMO radar using spectrum estimation theory," IET Radar, Sonar \& Navigation, vol. 11, no. 2, pp. 304-312, 2017.

[34] X. Hu, N. Tong, B. Song, S. Ding, and X. Zhao, "Joint sparsitydriven three-dimensional imaging method for multiple-input multiple-output radar with sparse antenna array," IET Radar, Sonar \& Navigation, vol. 11, no. 5, pp. 709-720, 2017.

[35] J. Xu, G. Liao, Y. Zhang, H. Ji, and L. Huang, "An adaptive range-angle-doppler processing approach for FDA-MIMO radar using three-dimensional localization," IEEE Journal of Selected Topics in Signal Processing, vol. 11, no. 2, pp. 309-320, 2017.

[36] X. Hu, N. Tong, X. He, and Y. Wang, "High resolution 3D imaging in MIMO radar with sparse array," Multidimensional Systems and Signal Processing, vol. 29, no. 2, pp. 745-759, 2018.

[37] Z. Jiao, C. Ding, X. Liang, L. Chen, and F. Zhang, "Sparse bayesian learning based three-dimensional imaging algorithm for off-grid air targets in MIMO radar array," Remote Sensing, vol. 10, no. 3, p. 369, 2018.

[38] K. Morimoto, T. Sakamoto, and T. Sato, "Accurate ultrawideband array radar imaging using four-dimensional unitary esprit," IEEE Access, vol. 7, pp. 161363-161374, 2019.

[39] M. Haardt and J. A. Nossek, "Unitary esprit: how to obtain increased estimation accuracy with a reduced computational burden," IEEE Transactions on Signal Processing, vol. 43, no. 5, pp. 1232-1242, 1995.

[40] M. D. Zoltowski, M. Haardt, and C. P. Mathews, "Closedform 2-D angle estimation with rectangular arrays in element space or beamspace via unitary esprit," IEEE Transactions on Signal Processing, vol. 44, no. 2, pp. 316-328, 1996.

[41] G. Zheng, B. Chen, and M. Yang, "Unitary esprit algorithm for bistatic MIMO radar," Electronics Letters, vol. 48, no. 3, p. 179, 2012.

[42] Emanuel and Q. Radoi, "Achieving superresolution by subspace eigenanalys is in multidimensional spaces," in Proceedings of the 11th European Signal Processing Conference, pp. 1-4, Toulouse, France, September 2002.

[43] X. Wang, M. Zhang, and Z. Jia, "Efficient cross-range scaling method via two-dimensional unitary esprit scattering center extraction algorithm," IEEE Geoscience and Remote Sensing Letters, vol. 12, no. 5, pp. 928-932, 2015.

[44] X. Wang, J. Zhao, and M. Zhang, "Super-resolution ISAR imaging via 2D unitary esprit," Electronics Letters, vol. 51, no. 6, pp. 519-521, 2015.

[45] J. Zhao, M. Zhang, X. Wang, Z. Cai, and D. Nie, “Threedimensional super resolution ISAR imaging based on $2 \mathrm{D}$ unitary ESPRIT scattering centre extraction technique," IET Radar, Sonar \& Navigation, vol. 11, no. 1, pp. 98-106, 2017.

[46] F. Liu, X. Wang, M. Huang, L. Wan, H. Wang, and B. Zhang, "A novel unitary esprit algorithm for monostatic FDA-MIMO radar,” Sensors, vol. 20, no. 3, p. 827, 2020. 
[47] T. Fu and X. Gao, "Simultaneous diagonalization with similarity transformation for non-defective matrices," in IEEE International Conference on Acoustics Speed and Signal Processing Proceedings, vol. 4, May 2006.

[48] R. Iferroudjene, K. Abed Meraim, and A. Belouchrani, "A new jacobi-like method for joint diagonalization of arbitrary non-defective matrices," Applied Mathematics and Computation, vol. 211, no. 2, pp. 363-373, 2009.

[49] D. Hutchison, T. Kanade, J. Kittler et al., "Joint eigenvalue decomposition using polar matrix factorization," in Latent Variable Analysis and Signal Separation, V. Vincent, V. Zarzoso, E. Moreau, R. Gribonval, and E. Vincent, Eds., Springer, Berlin, Germany, pp. 555-562, 2010.

[50] X. Luciani and L. Albera, "Joint eigenvalue decomposition of non-defective matrices based on the LU factorization with application to ICA," IEEE Transactions on Signal Processing, vol. 63, no. 17, pp. 4594-4608, 2015.

[51] R. Andre, T. Trainini, X. Luciani, and E. Moreau, "A fast algorithm for joint eigenvalue decomposition of real matrices," in Proceeding of the 23rd European Signal Processing Conference (EUSIPCO), pp. 1316-1320, IEEE, Nice, France, August 2015.

[52] H. Akaike, "A new look at the statistical model identification," IEEE Transactions on Automatic Control, vol. 19, no. 6, pp. 716-723, 1974.

[53] J. Rissanen, "Modeling by shortest data description," Automatica, vol. 14, no. 5, pp. 465-471, 1978.

[54] H.-T. Wu and C.-L. Chen, "A new gerschgorin radii based method for source number detection," in Proceedings of the Tenth IEEE Workshop on Statistical Signal and Array Processing, pp. 104-107, IEEE, Pocono Manor, PA, USA, August 2000.

[55] L. De Lathauwer, B. De Moor, and J. Vandewalle, "Computation of the canonical decomposition by means of a simultaneous generalized schur decomposition," SIAM Journal on Matrix Analysis and Applications, vol. 26, no. 2, pp. 295-327, 2004

[56] E. Rafael Balda, S. A. Cheema, A. Weiss, A. Yeredor, and M. Haardt, "Perturbation analysis of joint eigenvalue decomposition algorithms," in Proceedings of the IEEE International Conference on Acoustics, Speech and Signal Processing (ICASSP), pp. 3101-3105, IEEE, New Orleans, LA, USA, March 2017. 